\title{
Abnormalities of resting-state EEG in patients with prodromal and overt dementia with Lewy bodies: Relation to clinical symptoms
}

\author{
Maria Teresa Pascarelli ${ }^{a}$, Claudio Del Percio ${ }^{b}$, Maria Francesca De Pandis ${ }^{c}$, Raffaele Ferri ${ }^{a}$, Roberta Lizio ${ }^{\mathrm{d}}$, \\ Giuseppe Noce $^{\mathrm{d}}$, Susanna Lopez ${ }^{\mathrm{aa}}$, Marco Rizzo ${ }^{\mathrm{a}}$, Andrea Soricelli ${ }^{\mathrm{d}, \mathrm{e}}$, Flavio Nobili ${ }^{\mathrm{f}, \mathrm{g}}$, Dario Arnaldi ${ }^{\mathrm{f}, \mathrm{g}}$, \\ Francesco Famà ${ }^{\mathrm{g}}$, Francesco Orzi ${ }^{\mathrm{h}}$, Carla Buttinelli ${ }^{\mathrm{h}}$, Franco Giubilei ${ }^{\mathrm{h}}$, Marco Salvetti ${ }^{\mathrm{h}, \mathrm{i}}$, Virginia Cipollini ${ }^{\mathrm{h}}$, \\ Raffaella Franciotti ${ }^{\mathrm{j}}$, Marco Onofri ${ }^{\mathrm{j}}$, Peter Fuhr ${ }^{\mathrm{k}}$, Ute Gschwandtner ${ }^{\mathrm{k}}$, Gerhard Ransmayr ${ }^{\mathrm{l}}$, Dag Aarsland ${ }^{\mathrm{m}}$, \\ Lucilla Parnetti $^{\mathrm{n}}$, Lucia Farotti ${ }^{\mathrm{n}}$, Moira Marizzoni ${ }^{\circ}$, Fabrizia D'Antonio ${ }^{\mathrm{p}}$, Carlo De Lena ${ }^{\mathrm{p}}$, Bahar Güntekin ${ }^{\mathrm{q}}$, \\ Lutfu Hanoğlu ${ }^{r}$, Görsev Yener ${ }^{\mathrm{s}, \mathrm{z}}$, Derya Durusu Emek-Savaş ${ }^{\mathrm{t}}$, Antonio Ivano Triggiani ${ }^{\mathrm{u}}$, John Paul Taylor ${ }^{\mathrm{v}}$, \\ Ian McKeith ${ }^{v}$, Fabrizio Stocchi ${ }^{w}$, Laura Vacca ${ }^{w}$, Harald Hampel ${ }^{\mathrm{x}}$, Giovanni B. Frisoni ${ }^{\mathrm{o}, \mathrm{y}}$, Laura Bonanni ${ }^{\mathrm{j}}$, \\ Claudio Babiloni ${ }^{\mathrm{b}, \mathrm{c}, *}$
}

a Oasi Research Institute - IRCCS, Troina, Italy

${ }^{\mathrm{b}}$ Department of Physiology and Pharmacology “V. Erspamer”, Sapienza University of Rome, Rome, Italy

' San Raffaele of Cassino, Cassino, FR, Italy

' IRCCS SDN, Napoli, Italy

${ }^{\mathrm{e}}$ Department of Motor Sciences and Healthiness, University of Naples Parthenope, Naples, Italy

${ }^{\mathrm{f}}$ Clinica neurologica, IRCCS Ospedale Policlinico San Martino, Genova, Italy

${ }^{\mathrm{g}}$ Dipartimento di Neuroscienze, Oftalmologia, Genetica, Riabilitazione e Scienze Materno-infantili (DiNOGMI), Università di Genova, Italy

${ }^{\mathrm{h}}$ Department of Neuroscience, Mental Health and Sensory Organs, Sapienza University of Rome, Rome, Italy

${ }^{i}$ Neuromed: IRCCS Istituto Neurologico Mediterraneo (INM) Neuromed, 86077 Pozzilli, IS, Italy

${ }^{\mathrm{j}}$ Department of Neuroscience Imaging and Clinical Sciences and CESI, University G d'Annunzio of Chieti-Pescara, Chieti, Italy

${ }^{\mathrm{k}}$ Universitätsspital Basel, Abteilung Neurophysiologie, Petersgraben 4, 4031 Basel, Switzerland

${ }^{1}$ Department of Neurology 2, Med Campus III, Faculty of Medicine, Johannes Kepler University, Kepler University Hospital, Krankenhausstr. 9, A-4020 Linz, Austria

${ }^{\mathrm{m}}$ Department of Old Age Psychiatry, King's College University, London, UK

${ }^{n}$ Centre for Memory Disturbances, Lab of Clinical Neurochemistry, Section of Neurology, University of Perugia, Italy

${ }^{\circ}$ Laboratory of Alzheimer's Neuroimaging and Epidemiology, IRCCS Istituto Centro San Giovanni di Dio Fatebenefratelli, Brescia, Italy

${ }^{\mathrm{P}}$ Department of Human Neurosciences, Sapienza University of Rome, Italy

${ }^{\mathrm{q}}$ Department of Biophysics, International School of Medicine, Istanbul Medipol University, Istanbul, Turkey

${ }^{\mathrm{r}}$ Department of Neurology, School of Medicine, Istanbul Medipol University, Istanbul, Turkey

${ }^{s}$ Izmir Biomedicine and Genome Center, Dokuz Eylul University Health Campus, Izmir, Turkey

${ }^{\mathrm{t}}$ Department of Psychology and Department of Neurosciences, Dokuz Eylül University, Izmir, Turkey

${ }^{\mathrm{u}}$ Department of Clinical and Experimental Medicine, University of Foggia, Foggia, Italy

${ }^{\mathrm{v}}$ Institute of Neuroscience, Newcastle University, Newcastle, UK

${ }^{\mathrm{w}}$ Institute for Research and Medical Care, IRCCS San Raffaele Pisana, Rome, Italy

${ }^{\mathrm{x}}$ Department of Neurology, Institute of Memory and Alzheimer's Disease (IM2A), Brain and Spine Institute (ICM), François Lhermitte Building, France

${ }^{\mathrm{y}}$ Memory Clinic and LANVIE - Laboratory of Neuroimaging of Aging, University Hospitals and University of Geneva, Geneva, Switzerland

${ }^{\mathrm{z}}$ Department of Neurosciences, Institute of Health Sciences, Dokuz Eylul University, Izmir, Turkey

${ }^{\text {aa }}$ Department of Emergency and Organ Transplantation - Nephrology, Dialysis and Transplantation Unit, Aldo Moro University of Bari, Bari, Italy

\section{A R T I C L E I N F O}

\section{Article history:}

Accepted 7 September 2020

Available online 23 September 2020

\section{Keywords:}

Prodromal and overt dementia with Lewy

bodies (DLB)

\section{H I G H L I G H T S}

- Patients with prodromal-overt dementia with Lewy bodies (DLB) and visual hallucinations show highest parietal delta rhythms.

- DLB patients with the greatest cognitive deficits show highest posterior alpha rhythms.

- EEG rhythms are related to clinical manifestations in DLB patients.

\footnotetext{
* Corresponding author at: Department of Physiology and Pharmacology “V. Erspamer”, Sapienza University of Rome, P. le A. Moro 5, 00185 Rome, Italy.

E-mail address: claudio.babiloni@uniroma1.it (C. Babiloni).
} 
Mild cognitive impairment (MCI)

Resting state electroencephalographic (EEG) rhythms

Exact low-resolution brain electromagnetic source tomography (eLORETA)

Visual hallucinations

Rapid eye movement sleep behavior

disorders (RBD)

\begin{abstract}
A B S T R A C T
Objective: Here we tested if cortical sources of resting state electroencephalographic (rsEEG) rhythms may differ in sub-groups of patients with prodromal and overt dementia with Lewy bodies (DLB) as a function of relevant clinical symptoms.

Methods: We extracted clinical, demographic and rsEEG datasets in matched DLB patients $(\mathrm{N}=60)$ and control Alzheimer's disease $(A D, N=60)$ and healthy elderly (Nold, $N=60)$ seniors from our international database. The eLORETA freeware was used to estimate cortical rsEEG sources.

Results: As compared to the Nold group, the DLB and AD groups generally exhibited greater spatially distributed delta source activities (DLB > AD) and lower alpha source activities posteriorly $(A D>D L B)$. As compared to the DLB "controls", the DLB patients with (1) rapid eye movement (REM) sleep behavior disorders showed lower central alpha source activities ( $<$ 0.005); (2) greater cognitive deficits exhibited higher parietal and central theta source activities as well as higher central, parietal, and occipital alpha source activities ( $\mathrm{p}<0.01$ ); (3) visual hallucinations pointed to greater parietal delta source activities ( $\mathrm{p}<0.005)$.

Conclusions: Relevant clinical features were associated with abnormalities in spatial and frequency features of rsEEG source activities in DLB patients.

Significance: Those features may be used as neurophysiological surrogate endpoints of clinical symptoms in DLB patients in future cross-validation prospective studies.
\end{abstract}

(C) 2020 International Federation of Clinical Neurophysiology. Published by Elsevier B.V. All rights

\section{Introduction}

Patients with Lewy body dementia (LBD) are characterized by progressive neurodegeneration of cortical and sub-cortical reticular neural systems due to accumulation of intracellular Lewy bodies and neurofibrillary tangles (Weisman et al., 2007). Of consequence, clinical phenotype of LBD includes motor, behavioral and psychiatric dysfunctions as visual hallucinations ( $>80 \%$, McKeith et al., 2017), fluctuation of cognitive disorders, and disturbances of rapid eye movement (REM) sleep (McKeith et al., 1996, 2017; Donaghy \& McKeith, 2014).

A reliable diagnosis is allowed only in the late stage of the disease as these mentioned clinical manifestations in part overlap with those of dementia due to Parkinson's disease (PDD) and Alzheimer's disease (ADD).

The diagnostic process may imply the clinical and instrumental re-evaluation of ADD, PDD, and LBD patients over time every 6 or 12 months.

rsEEG markers were tested in several LBD studies as indexes of brainstem-thalamus-cortical neural synchronization mechanisms underpinning the regulation of vigilance in quiet wakefulness (Fünfgeld, 1995; Bosboom et al., 2009, 2006; Bonanni et al., 2008; Serizawa et al., 2008; Kamei et al., 2010; Pugnetti et al., 2010; Melgari et al., 2014; Caviness et al., 2016).

Main results showed slower rsEEG activity at delta and theta frequencies (respectively $<4$ and $4-7 \mathrm{~Hz}$ ) and a reduction at alpha frequencies (8-12 Hz) in LBD patients respect to normal healthy elderly (Nold) individuals (Bonanni et al., 2008; Jackson et al., 2008).

Here we used a methodological approach described in a previous article of our Consortium (Babiloni et al., 2020b). That description is reported between quotation markers in the following: "(1) the estimation of rSEEG cortical sources by the popular exact lowresolution brain electromagnetic tomography (eLORETA) freeware (Pascual-Marqui, 2007) and (2) the definition of delta, theta, and alpha frequency bands on individual basis using the so-called individual alpha frequency (IAF) peak as a reference in the rsEEG power density spectrum averaged across all electrodes" (Babiloni et al., 2020b). Main results of this approach can be summarized as follows: compared to Nold subjects, LBD showed lower activation in occipital, parietal, and temporal cortical sources of alpha rhythms around the IAF, associated with an increase in topographically widespread delta source activities (Babiloni et al., 2017a,b, 2018a,b,c). Such abnormalities in the delta and alpha source activities were intermediate between those observed in ADD and PDD patients, where PDD and ADD showed maximum abnormalities in delta and alpha, respectively. Similar results were observed in those patients even at the prodromal stage of mild cognitive impairment. From a neurophysiological point of view, that abnormal reduction in alpha source activities may reflect a background cortical overexcitation in quiet wakefulness (Pfurtscheller and Lopes da Silva, 1999). Furthermore, those abnormalities in both delta and alpha source activities in resting state conditions may reflect alterations in the neurophysiological oscillatory systems regulating vigilance in quiet wakefulness. To support this tentative explanation, previous studies investigating rsEEG rhythms have reported that sleep deprivation induces an increase in posterior cortical delta rhythms and a reduction in widespread alpha rhythms recorded in healthy adults resting in quiet wakefulness, while the acute administration of a pharmacological agent enhancing the vigilance (i.e., Modafinil) partially recovers these effects (Chapotot et al., 2003; James et al., 2011; Bodenmann et al., 2009; Saletu et al., 2004, 2007). The relationship between cortical delta and alpha rhythms in the resting state condition and the neurophysiological regulation of quiet vigilance makes these rsEEG markers extremely interesting for investigating the clinical neurophysiology of fluctuations of cognitive functions, visual hallucinations, and rapid eye movement (REM) sleep behavior disorders in LBD patients.

In the present retrospective and explorative study, we used the mentioned methodological approach on an international database to test the hypothesis that in DLB patients, cortical sources of rsEEG rhythms frequencies may show differences in relation to relevant clinical features such as fluctuation of cognitive deficits, REM behavioral disorders, and visual hallucinations. Delta and alpha source activities were the main variables of interest based on our previous field studies (Babiloni et al., 2017a,b, 2018a,b,c), while theta, beta, and gamma source activities were used for other purposes. On the one hand, they were used to control the specificity of the effects at delta and alpha source activities. On the other hand, temporal and frontal beta and gamma source activities showing relatively low and decreasing values as a function of frequencies represent an important benchmark of the quality of rsEEG recording, preliminary data analysis, and source estimation in subjects without major muscle tensions in eyes-closed resting state conditions. 
In the experimental design of the present study, cortical sources of rsEEG rhythms were compared between matched sub-groups of patients with prodromal and overt dementia with Lewy Bodies (DLB) stratifying them based on relevant clinical features, namely those having high vs. low global cognitive status, the presence or absence of visual hallucinations, and the presence or absence of REM sleep behavior disorders. In this design, Nold and AD seniors were used as control groups to address the specificity of the effects.

\section{Materials and methods}

\subsection{Subjects}

In the present retrospective and observational study, clinical and rsEEG data were extracted from an international archive formed by the Clinical Units and E-DLB Consortia. Specifically, the DLB group $(\mathrm{N}=60)$ was formed by 42 patients with dementia and 18 patients with its prodromal stage, namely the condition of mild cognitive impairment ( $\mathrm{MCI})$ as defined in the following section. As mentioned above, the DLB group was matched for age, gender, and education with groups of $A D$ patients $(N=60 ; 20$ patients with dementia, $A D D$, and 40 patients with mild cognitive impairment, ADMCI) and Nold subjects $(\mathrm{N}=60)$, whose data were extracted from the same database for control purposes. All DLB and $A D$ patients performed the rsEEG recording within one year from the clinical diagnosis. Only DLB and AD patients whose diagnosis has been confirmed at almost one visit of follow up have been considered for this study. DLB group was further divided in 3 pairs sub-groups according to clinical criteria for statistical comparison. Specifically, DLB group was divided in DLB VH+ and DLB VHaccording to the presence or not of visual hallucination; same logic was applied to extract DLB RBD+ and DLB RBD - subgroups respectively with and without REM sleep behavior disorders; the median value of MMSE defined DLB sub-groups with lowest (DLB MMSE-) and highest (DLB MMSE+) global cognitive deficits. Each pair of DLB sub-groups was matched for age, gender, and education. UPDRS III motor score and pharmacological therapies (i.e., levodopa, acetylcholinesterase inhibitors -AChEIs, and antipsychotics drugs) were also considered to avoid any confounding effects.

Local institutional Ethics Committee approved the present observational study. All experiments were performed with the informed and overt consent of each participant or caregiver, in line with the Code of Ethics of the World Medical Association (Declaration of Helsinki) and the standards established by the local Institutional Review Board.

Table 1 summarizes demographic (i.e., age, gender, and education) and clinical (i.e., mini mental state evaluation, MMSE score; visual hallucinations, VH; Unified Parkinson Disease Rating ScaleIII, UPDRS III score) data of those Nold, AD, DLB groups and pharmacological therapies of DLB sub-groups. To test the presence or absence of statistically significant differences $(p<0.05)$ among demographic and clinical variables of the Nold, AD and DLB groups or DLB subgroups the following test were used: ANOVA for age and education; Freeman-Halton for gender and Kruskal-Wallis for MMSE score, Fisher's test for visual hallucinations and t-test for UPDRS score. Fisher's test was also used to evaluate the statistical difference between pairs of the DLB-subgroups in the assumption of levodopa, AChEIs, and antipsychotics drugs.

\subsection{Diagnostic criteria}

The diagnosis of the probable DLB was carried out according to the consensus guidelines (McKeith et al., 2005, 2017). Twenty-six out of 60 DLB patients performed DaTSCAN to confirm the diagno- sis of probable DLB. The clinical features of DLB were detected as follows:

the Neuropsychiatric Inventory (NPI) item-2 tested the occurrence frequency and the severity of hallucinations (Cummings et al., 1994); the severity of the frontal dysfunction was investigated by Frontal Assessment Battery (FAB) (Dubois et al., 2000); the presence and severity of the cognitive fluctuations by Clinician Assessment of Fluctuations (Walker et al., 2000a,b); extrapyramidal signs were evaluated by Unified Parkinson Disease Rating Scale-III (UPDRS-III) (Fahn et al., 1987); minimal International Classification of Sleep Disorders criteria (1992) were used to determinate the presence and/or absence of rapid eye movement (REM) sleep behavior disorder (RBD).

DLBMCI subjects underwent further different neuropsychological tests to evaluate the status of MCI (Donaghy et al., 2018).

The diagnosis of ADD and ADMCI has been described in a previous reference article of our Consortium (Babiloni et al., 2020b). That description is reported between quotation marks in the following paragraph: "The $A D D$ and $A D M C I$ were diagnosed according to the DSM-IV-TR and the National Institute of Neurological Disorders and Stroke-Alzheimer Disease and Related Disorders (NINCDS-ADRDA) working group (McKhann et al., 1984). Exclusion criteria for the ADMCI patients were other significant neurological, systemic or psychiatric illness, mixed dementing diseases, enrolment in a clinical trial with experimental drugs, the use of antidepressant drugs with anticholinergic side effects, high dose of neuroleptics or chronic sedatives or hypnotics, antiparkinsonian medication and the use of narcotic analgesics" (Babiloni et al., 2020b).

All Nold subjects underwent physical, cognitive (i.e., MMSE, 15item Geriatric Depression Scale-GDS, Brown and Schinka, 2005) and neurological examinations to exclude previous or present neurological or psychiatric disease. Further exclusion criteria were the presence of depression (detected with a GDS score higher than 5), use of chronic psychoactive drugs, and chronic systemic illnesses (e.g. diabetes mellitus).

\subsection{RSEEG recordings and preliminary data analysis}

EEG recordings were performed in the late morning to minimize drowsiness. Instructions kindly asked subjects to have a moderate breakfast and refrain from the assumption of psychoactive drugs (e.g., antidepressant, benzodiazepine) and other psychostimulants (e.g., coffee, tea) in the morning of the rsEEG recording. Furthermore, subjects were kindly asked to inform experimenters about the quality of sleep the night before the EEG recording and any other event possibly perturbing physical and mental status in a way incompatible with the experiment.

Instructions also dealt with subjects' behavioral state during the rsEEG recording. All subjects were kindly asked to remain in a standard resting state condition. In this condition, they had to keep muscle relaxation without movements/talking and maintain their mind awake and freely wandering without systematic goaloriented mentalization.

On-going rsEEG activity was recorded in a quiet and dimly lighted room for at least 5 minutes while subjects seated on a comfortable half reclined armchair with eyes closed in the mentioned standard resting state condition. All digital EEG recording systems were certified for clinical use in hospitals such as EBNeuro, Micromed, Brain vision, etc. The procedures for the EEG recording and preliminary data analysis were described in a previous reference article of our Consortium (Babiloni et al., 2019). That description is reported between quotation marks in the following paragraphs:

"The rsEEG data were recorded with a sampling frequency of 256$1024 \mathrm{~Hz}$ and related antialiasing bandpass between $0.01 \mathrm{~Hz}$ and $100 \mathrm{~Hz}$. Nineteen scalp electrodes positioned according to the 10-20 System (i.e. Fp1, Fp2, F7, F3, Fz, F4, F8, T3, C3, Cz, C4, T4, T5, P3, Pz, 
Table 1

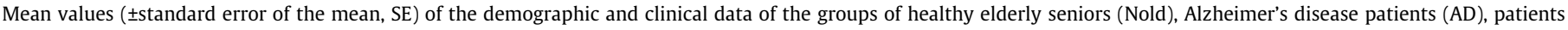

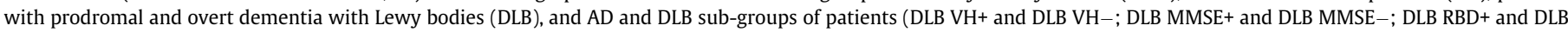

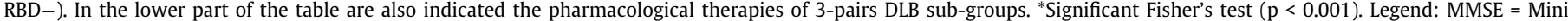

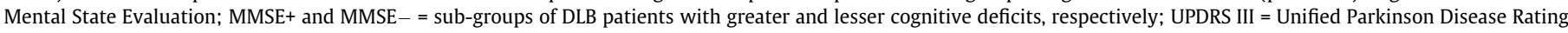

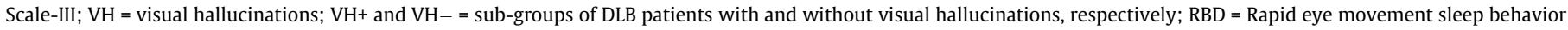
disorder; RBD+ and RBD- = sub-groups of DLB patients with and without rapid eye movement sleep behavior disorder, respectively.

\begin{tabular}{|c|c|c|c|c|c|c|}
\hline \multicolumn{7}{|c|}{ Demographic and clinical characteristics } \\
\hline Group/N & Age & Gender (F/M) & Education & MMSE score (Nold > AD, DLB) & UPDRS III & $\mathrm{VH}$ \\
\hline Nold (60) & $74.4( \pm 0.7 \mathrm{SE})$ & $32 / 28$ & $9.4( \pm 0.5 \mathrm{SE})$ & $28.4( \pm 0.1 \mathrm{SE})$ & - & - \\
\hline $\begin{array}{l}\mathrm{AD} \\
\qquad(60 ; 20 \mathrm{ADD}, 40 \mathrm{ADMCI})\end{array}$ & $75.2( \pm 0.5 \mathrm{SE})$ & $35 / 25$ & $9.6( \pm 0.6 \mathrm{SE})$ & $22.6( \pm 0.5 \mathrm{SE})$ & - & - \\
\hline $\begin{array}{l}\text { DLB } \\
\qquad(60 ; 42 \text { DLB, } 18 \text { DLBMCI })\end{array}$ & $76.2( \pm 0.7 \mathrm{SE})$ & $27 / 33$ & $8.5( \pm 0.6 \mathrm{SE})$ & $20.8( \pm 0.6 \mathrm{SE})$ & $20.5( \pm 1.6 \mathrm{SE})$ & 40 \\
\hline $\begin{array}{l}\text { DLB VH+ } \\
\qquad(40 ; 29 \text { DLB, } 11 \text { DLBMCI })\end{array}$ & $75.5( \pm 0.9 \mathrm{SE})$ & $18 / 22$ & $8.7( \pm 0.7 \mathrm{SE})$ & $20.3( \pm 0.7 \mathrm{SE})$ & $20.8( \pm 2.0 \mathrm{SE})$ & 40 \\
\hline $\begin{array}{l}\text { DLB VH- } \\
\qquad(17 ; 6 \text { DLB, } 11 \text { DLBMCI })\end{array}$ & $77.5( \pm 1.3 \mathrm{SE})$ & $8 / 9$ & $7.6( \pm 1.0 \mathrm{SE})$ & $21.6( \pm 1.1 \mathrm{SE})$ & $20.9( \pm 3.4 \mathrm{SE})$ & 0 \\
\hline $\begin{array}{l}\mathrm{AD} \\
\qquad(25 ; 15 \mathrm{ADD}, 10 \mathrm{ADMCI})\end{array}$ & $76.7( \pm 0.8 \mathrm{SE})$ & $15 / 10$ & $9.9( \pm 0.9 \mathrm{SE})$ & $19.3( \pm 0.7 \mathrm{SE})$ & - & - \\
\hline $\begin{array}{l}\text { DLB MMSE+ } \\
\qquad(26 ; 24 \text { DLB, } 2 \text { DLBMCI })\end{array}$ & $76.5( \pm 1.0 \mathrm{SE})$ & $13 / 13$ & $7.4( \pm 0.8 \mathrm{SE})$ & $16.9( \pm 0.6 \mathrm{SE})$ & $23.6( \pm 2.7 \mathrm{SE})$ & 19 \\
\hline $\begin{array}{l}\text { DLB MMSE- } \\
\quad(26 ; 15 \text { DLB, } 11 \text { DLBMCI })\end{array}$ & $75.7( \pm 1.0 \mathrm{SE})$ & $9 / 17$ & $9.4( \pm 0.8 \mathrm{SE})$ & $24.7( \pm 0.4 \mathrm{SE})$ & $19.0( \pm 2.3 \mathrm{SE})$ & 15 \\
\hline $\begin{array}{l}\text { DLB RBD+ } \\
\qquad(42 ; 28 \text { DLB, } 14 \text { DLBMCI })\end{array}$ & $76.1( \pm 0.8 \mathrm{SE})$ & $15 / 27$ & $8.6( \pm 0.8 \mathrm{SE})$ & $21.5( \pm 0.7 \mathrm{SE})$ & $19.4( \pm 1.9 \mathrm{SE})$ & 26 \\
\hline $\begin{array}{l}\text { DLB RBD- } \\
\qquad(14 ; 12 \text { DLB, } 2 \text { DLBMCI })\end{array}$ & $76.5( \pm 1.6 \mathrm{SE})$ & $9 / 5$ & $8.7( \pm 0.6 \mathrm{SE})$ & $19.4( \pm 0.9 \mathrm{SE})$ & $22.9( \pm 3.4 \mathrm{SE})$ & 10 \\
\hline \multicolumn{7}{|l|}{ Pharmacological therapies } \\
\hline & Levodopa & & AChEI & & Psychoactive drugs & \\
\hline DLB VH+ & $48 \%$ & & $45 \%$ & & $45 \%$ & \\
\hline DLB VH- & $59 \%$ & & $29 \%$ & & $35 \%$ & \\
\hline DLB MMSE+ & $50 \%$ & & $42 \%$ & & $46 \%$ & \\
\hline DLB MMSE- & $62 \%$ & & $35 \%$ & & $23 \%$ & \\
\hline DLB RBD+ & $62 \%$ & & $26 \%$ & & $29 \% *$ & \\
\hline DLB RBD- & $29 \%$ & & $64 \%$ & & $71 \% *$ & \\
\hline
\end{tabular}

P4, T6, 01, and 02) were used with respect to a cephalic ground (i.e., frontal) and an extracephalic electrode reference (i.e., linked auricular or mastoid). Electrodes impedances were kept below $5 \mathrm{KOhm}$. Bipolar vertical and horizontal electrooculographic (EOG), and one-channel electrocardiographic signals were also acquired (256-1024 Hz) for artifact detection and off-line correction (when possible)" (Babiloni et al. 2019). During the EEG recording, the experimenters did control subject's behavioral condition and on-going rsEEG traces, to help subject to keep the adequate level of vigilance (i.e., avoiding drowsiness and sleep onset).

"The recorded rsEEG data were divided into epochs of 2 seconds and analyzed off-line. The epochs affected by any physiological (ocular/blinking, muscular, head movements) or non-physiological (bad contact electrode-scalp) artifacts were preliminarily identified by an automatic computerized procedure (Moretti et al., 2003). Furthermore, two independent experimenters manually checked and (dis)confirmed the artifact-free rsEEG epochs, before successive analyses. Specifically, they controlled for the presence of ocular and blinking artifacts based on EOG signals, while muscular and head artifacts were recognized by analyzing EEG signals. Moreover, head artifacts were detected by a sudden and great increase in amplitude of slow EEG waves in all scalp electrodes. Finally, muscle artifacts were recognized observing the effects of several frequency bandpass filters in different ranges and by the inspection of EEG power density spectra. Indeed, muscle tension is related to unusually high and stable values of EEG power density from 30 to $100-150 \mathrm{~Hz}$, which contrast with the typical declining trend of EEG power density from $25 \mathrm{~Hz}$ onward" (Babiloni et al., 2019).

Artifact-free rsEEG epochs were off-line frequency-band passed at $0.1-45 \mathrm{~Hz}$ and down sampled, when appropriate, to make the sampling rate of all rsEEG datasets in patients and control seniors equal to $128 \mathrm{~Hz}$. For sake of harmonization of the datasets, all recorded rsEEG data were re-referenced to the common average reference.

\subsection{Spectral analysis of the rsEEG epochs}

The procedures for the EEG spectral analysis were described in a previous reference article of our Consortium (Babiloni et al., 2019). That description is reported between quotation marks in the following paragraphs:

"A standard digital FFT-based power spectrum analysis (Welch technique, Hanning windowing function, no phase shift) computed the power density of scalp rsEEG rhythms with $0.5 \mathrm{~Hz}$ of frequency resolution.

According to a previous study of our group (Moretti et al., 2004), the frequency bands of interest were individually identified based on the following frequency landmarks, namely the transition frequency (TF) and the individual alpha frequency peak (IAF). In the EEG power density spectrum, the TF marks the transition frequency between the theta and alpha bands, defined as the minimum of the rsEEG power density between 3 and $8 \mathrm{~Hz}$ (between the delta and the alpha power peak). The IAF is defined as the maximum power density peak between 6 and $14 \mathrm{~Hz}$. These frequency landmarks were previously well described by Dr Wolfgang Klimesch (Klimesch, 1999, 1996; Klimesch et al., 1998).

The TF and IAF were individually computed for each subject involved in the present study. Based on the TF and IAF, we estimated the frequency band range for each subject as follows: delta from $T F$ $-4 \mathrm{~Hz}$ to $\mathrm{TF}-2 \mathrm{~Hz}$, theta from $\mathrm{TF}-2 \mathrm{~Hz}$ to $T F$, low-frequency alpha band (alpha 1 and alpha 2) from TF to IAF, and high-frequency alpha band (or alpha 3) from IAF to IAF $+2 \mathrm{~Hz}$. The other bands were defined 
based on standard fixed frequency ranges, namely beta 1 from 14 to $20 \mathrm{~Hz}$, beta 2 from 20 to $30 \mathrm{~Hz}$, and gamma from 30 to $40 \mathrm{~Hz}$. The alpha 1 and alpha 2 bands were computed for each subject as follows: alpha 1 from TF to the midpoint of the TF-IAF range and alpha 2 from this midpoint to IAF" (Babiloni et al., 2019).

\subsection{Cortical sources of rsEEG epochs as computed by eLORETA}

The procedures for the EEG cortical source estimations were described in a previous reference article of our Consortium (Babiloni et al., 2019). That description is reported between quotation marks in the following paragraphs:

"We used the official freeware tool called exact LORETA (eLORETA) for the linear estimation of the cortical source activity generating scalp-recorded rsEEG rhythms (Pascual-Marqui, 2007). The present implementation of eLORETA uses a head volume conductor model composed of the scalp, skull, and brain. In the scalp compartment, exploring electrodes can be virtually positioned to give EEG data as an input to the source estimation (Pascual-Marqui, 2007). The brain model is based on a realistic cerebral shape taken from a template typically used in the neuroimaging studies, namely that of the Montreal Neurological Institute (MNI152 template). The eLORETA freeware solves the so-called EEG inverse problem estimating "neural" current density values at any cortical voxel of the mentioned head volume conductor model. The solutions are computed rsEEG frequency binby-frequency bin.

The input for this estimation is the EEG spectral power density computed at 19 scalp electrodes. The output is the electrical brain source space formed by 6239 voxels with $5 \mathrm{~mm}$ resolution, restricted to the cortical grey matter of the head volume conductor model. An equivalent current dipole is located in each voxel. For each voxel, the eLORETA package provides the Talairach coordinates, the lobe, and the Brodmann area (BA).

In line with the general low spatial resolution of the present EEG methodological approach (i.e., 19 scalp electrodes), we performed a regional analysis of the eLORETA solutions. For this purpose, we collapsed the eLORETA solutions within frontal, central, temporal, parietal, occipital, and limbic macro-regions (ROIs) considered separately. Table 1 in Supplementary Materials reports the list of the $B A s$ used for the ROIs considered in the present study. Of note, the main advantage of the regional analysis of eLORETA solutions was that we could disentangle the rsEEG source activity in contiguous cortical areas. For example, the rSEEG source activity in the occipital ROI was disentangled from that estimated in the parietal and temporal ROIs, etc. This was made possible by the fact that eLORETA solves the linear inverse problem by considering (at least in part) the effects of the head as a volume conductor. In contrast, the solutions of rsEEG power density computed at a parietal scalp electrode reflect the contribution of source activities not only of the underlying parietal cortex but also of surrounding occipital and temporal cortices.

For the present eLORETA cortical source estimation, a frequency resolution of $0.5 \mathrm{~Hz}$ was used, namely, the maximum frequency resolution allowed by the use of 2-s artifact-free EEG epochs" (adapted from Babiloni et al., 2019).

\subsection{Statistical analysis of the rSEEG source activity at the group level}

The statistical analyses were performed by the commercial tool STATISTICA 10 (StatSoft Inc., www.statsoft.com) to test the control and working hypotheses. In all the statistical analyses, to limit false positive findings, the procedure was based on four steps. In the first step, the regional normalized rsEEG source solutions were Log-10 transformed to make them Gaussian before to be used as inputs to the ANOVA designs as a dependent variable $(p<0.05)$. Of note, we did not use non-parametric statistical analyses on untransformed rsEEG source solutions as the main hypotheses of the present study required a factorial design with 3 ways (e.g., Group, Band, and ROI) unsupported by those analyses. In the second step, we compared rsEEG source activity among the groups of interest with an ANOVA design $(p<0.01)$. In the third step, if the ANOVA showed significant effects, we performed planned post-hoc comparisons of rsEEG source activity among the groups of interest $(p<0.01)$. In the fourth step, if the post-hoc tests showed significant differences among the groups of interest, we controlled if the distribution of the rsEEG source variables showing those differences were characterized by outliers, possibly explaining the effects. The outliers were controlled within any group, separately, by the iterative (leave-one-out) Grubbs' test (GraphPad Software, Inc, California, USA) at the arbitrary conservative threshold of $\mathrm{p}<0.001$.

More specifically, the regional normalized rsEEG source solutions were Log-10 transformed and used as inputs to the ANOVA designs as a dependent variable $(p<0.05)$. The KolmogorovSmirnov test (null hypothesis of non-Gaussian distributions rejected at $\mathrm{p}<0.05$ ) was used to confirm that those regional normalized eLORETA solutions approximated to Gaussian distributions. Duncan test was used for post-hoc comparisons with a liberal statistical threshold chosen in line with the exploratory nature of the present retrospective study $(p<0.01)$. The following control analysis procedure has been recently described in a reference article of our Consortium (Babiloni et al., 2020b). That description has been adapted and reported between quotation marks in the following paragraphs:

"To cross-validate the above rSEEG (eLORETA) source differences between DLB sub-groups, we performed an independent component analysis (ICA) of scalp rsEEG rhythms at the group level. Specifically, we orthogonalized the recorded rSEEG time series at the scalp electrodes. For this analysis, we used the ICA toolbox of the eLORETA freeware platform (Pascual-Marqui, 2007; Kovacevic and McIntosh, 2007; Diaconescu et al., 2008). This toolbox computed the orthogonal independent components from scalp rsEEG rhythms recorded in all DLB and Nold participants as a whole group. Based on this procedure, each participant was associated with his/her respective individual independent components, each component being represented by time series derived from scalp recorded EEG rhythms.

Afterwards, for each participant, we computed the power density spectra from the time series explaining $>80 \%$ of the total variance of scalp-recorded rsEEG rhythms in that participant. Those spectral values were compared in the DLB patients' sub-groups characterized by lowest vs. highest global cognitive performance, the presence vs. the absence of visual hallucinations, and the presence vs. absence of REM sleep behavior disorders. Specifically, the following procedure was performed for each participant and independent component (i.e., considering the first five independent components, which explained $>80 \%$ of the total variance).

(1) The power density computed from the time series of a given component was normalized averaging values across all frequencies (i.e., $0.5-45 \mathrm{~Hz}$ ). The resulting mean value was used to normalize the power density of those time series at any frequency bin from 0.5 to $45 \mathrm{~Hz}$.

(2) All values of the normalized power density within each frequency band of interest were averaged to obtain the frequency band values (i.e., delta, theta, alpha 1, alpha 2, alpha 3, beta 1, beta 2 , and gamma).

(3) The normalized power density values were Log-10 transformed to make them Gaussian before subsequent parametric statistical analyses.

As a further step, fifteen ANOVAs (5 independent components for 3 clinical variables such as cognitive status, the presence/absence of visual hallucinations, and the presence/absence of REM sleep behavior 
disorders) were computed using the normalized (Log-10 transformed) power density values of the independent components as a dependent variable $(p<0.05)$.

Concerning the global cognitive status (i.e., MMSE score), five ANOVAs (one for each independent component) evaluated the hypothesis that the normalized power density of the independent components may differ as a function of that status in two sub-groups of DLB patients (DLB-MMSE- vs. DLB-MMSE+) and the Nold group. The ANOVA factors were Group (Nold, DLB-MMSE-, and DLB-MMSE+) and Band (from delta to gamma) $(p<0.001)$.

Concerning REM sleep behavior disorders, five ANOVAs (one for each independent component) evaluated the hypothesis that the normalized rsEEG power density of the independent components may differ as a function of those deficits in two sub-groups of DLB patients (DLB-RBD-vs. DLB-RBD+) and the Nold group. The ANOVA factors were Group (Nold, $D L B-R B D-$, and $D L B-R B D+$ ) and Band (from delta to gamma).

Concerning visual hallucinations, five ANOVAs (one for each independent component) evaluated the hypothesis that the normalized rsEEG power density of the independent components may be related to those symptoms in two sub-groups of DLB patients (DLB-VH-vs. $D L B-V H+)$ and the Nold group. The ANOVA factors were Group (Nold, $D L B-V H-$, and $D L B-V H+$ ) and Band (from delta to gamma)" (adapted from Babiloni et al., 2020b).

\subsection{Analysis of significant rsEEG source activities at the individual level}

The rsEEG source activities showing statistically significant differences ( $p<0.01$ ) between the DLB sub-groups were used as an input to the present analysis performed at the individual level. Again, the exploratory nature of the present study was reflected in the use of a liberal statistical threshold $(p<0.05)$ to characterize the most interesting rsEEG source activities for future prospective and longitudinal studies in DLB patients. This analysis was performed by the following tests.

First, the nonparametric Spearman test $(\mathrm{p}<0.05)$ was used to evaluate the correlation between the MMSE score (i.e., a rank scale not usable with parametric tests) and rsEEG source activities (i.e., regional normalized eLORETA solutions) showing statistically significant differences $(\mathrm{p}<0.01)$ between the DLB MMSE- and DLB MMSE+ groups.

Second, the results of the Spearman test were cross validated by computing a Partial Least Square (PLS) regression (Statistica 10). The PLS analysis tested the hypothesis that in the DLB patients, those significant rsEEG source activities may be related to the MMSE score $(p<0.05)$. In this respect, a principal component analysis (PCA) derived principal components from the significant rsEEG source activities as predictors, while MMSE scores were used as a dependent variable $(\mathrm{p}<0.05)$. The relevance of the principal components was expressed by two parameters, namely the "power" and the "independent variable importance". The "power" was defined as 1) the proportion of variability accounted for in the independent variables (i.e., EEG source activity) and 2) their variable importance in the projection (VIP) on the PLS model. The "power" was calculated using to the following formula:

Power $=1-\frac{S V j}{S V 0 j}$ es

where $S V_{j}$ is the residual standard variation of the jth variable and $S V 0 j$ is its initial standard deviation. In this frame, VIP is like the mentioned "power" but further weighted by the variability accounted for by the computed principal components.

Third, the rsEEG source activities showing differences between DLB sub-groups were used as an input for the classification of sin- gle individuals by the computation of the receiver operating characteristic (ROC; DeLong et al., 1988) curves, implemented in GraphPad software (GraphPad Software, Inc, California, USA). The following indexes measured the performance of the binary classification in one of the two groups: (1) Sensitivity as the rate of DLB patients of one group (e.g., the first group in the above pairs) correctly classified in the discrimination with the individuals of the other group (i.e., "true positive rate" in the signal detection theory); (2) Specificity as the rate of DLB patients of the other group correctly classified (i.e., "true negative rate" in the signal detection theory); (3) Accuracy as the weighted average of the sensitivity and specificity values; and (4) the Area under the ROC (AUROC) curve, considered as a major reference index of the global classification accuracy. ROC curve values $>0.7$ were considered as promising in future cross-validation studies.

\section{Results}

3.1. Statistical comparison of the rsEEG source activities in the Nold, $A D$, and $D L B$ groups

Table 2 reports the mean values of TF and IAF for the three groups (i.e., Nold, AD, and $\mathrm{DLB}$ ), together with the results of the statistical comparisons between the groups (ANOVA, $\mathrm{p}<0.05$ ). The mean TF was greater $(F=26.7, p<0.00001)$ in the Nold and AD than the DLB group ( $<<0.0001$ ); (2) the mean IAF was greater $(\mathrm{F}=39.6, \mathrm{p}<0.00001)$ in the Nold and AD than the DLB group $(p<0.0001)$. These findings stress the importance to use the TF and IAF in the determination of the delta to alpha frequency bands in the studies focused on AD and DLB patients.

Fig. 1 shows the grand average of regional rsEEG source activities (i.e., regional normalized eLORETA solutions log10 transformed) relative to a statistically significant ANOVA interaction effect $(F=7.8 ; p<0.00001)$ among the factors Group (Nold, AD, and DLB), Band (delta, theta, alpha 1, alpha 2, alpha 3, beta 1, beta 2, and gamma), and ROI (frontal, central, parietal, occipital, temporal, and limbic). Compared to the Nold group, the AD and DLB groups showed a substantial decrease of the eLORETA solutions in posterior (i.e. parietal and occipital) alpha 2 and alpha 3 sources. This effect was higher in the AD than the DLB group. Furthermore, the AD and DLB groups showed a substantial increase of the eLORETA solutions in central, parietal, occipital and temporal delta sources. This effect was higher in the DLB than the AD group.

The Duncan planned post-hoc testing showed that the discriminant pattern Nold $>$ DLB $>$ AD was fitted by parietal and occipital alpha 2 and alpha $3(\mathrm{p}<0.00001)$. Furthermore, the discriminant source pattern DLB > AD > Nold was fitted by central, parietal, occipital and temporal delta sources and temporal theta sources ( $p<0.00001$ ). Of note, these findings were not due to outliers from those individual eLORETA solutions, as shown by Grubbs' test with an arbitrary threshold of $\mathrm{p}<0.0001$ (see Fig. 1 in Supplementary Materials).

3.2. Statistical comparison of rsEEG source activities in the Nold, $A D$, $\mathrm{DLB} V \mathrm{H}-$, and $\mathrm{DLB} \mathrm{VH}+$ sub-groups

Table 2 reports the mean values of TF and IAF for the four subgroups (i.e., Nold, AD, DLB VH-, and DLB VH+), together with the results of the statistical comparisons between them (ANOVA, $\mathrm{p}<0.05)$. The mean TF was greater $(\mathrm{F}=16.9, \mathrm{p}<0.0001)$ in the Nold ( $\mathrm{p}<0.05$ ) than the AD, DLB VH- and DLB VH+ sub-groups; the mean IAF was greater $(F=25.3, p<0.0001)$ in the Nold and AD $(\mathrm{p}<0.0001$ ) than DLB VH- and DLB VH+ sub-groups.

Fig. 2 shows the grand average of regional rsEEG source activities (i.e., regional normalized eLORETA solutions $\log 10$ trans- 
Table 2

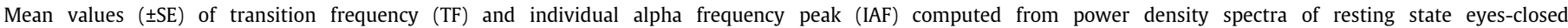

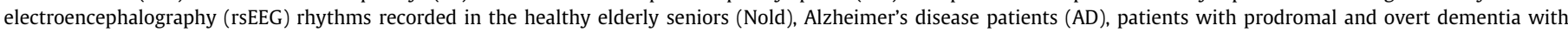

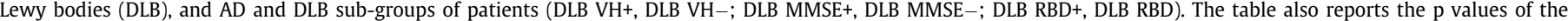

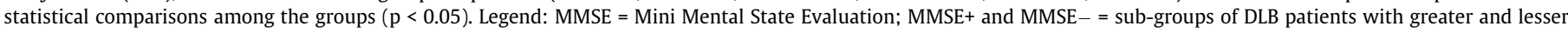

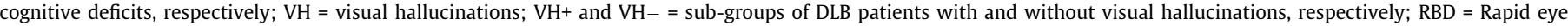

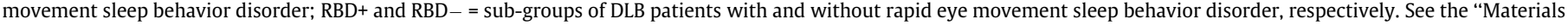
and Methods" (2.4 Spectral analysis of the rsEEG epochs) for the description of the procedure to determine TF and IAF.

\begin{tabular}{|c|c|c|c|c|c|}
\hline & $\mathrm{N}$ & $\mathrm{TF}$ & Statistical analysis for TF (ANOVA) & IAF & Statistical analysis for IAF (ANOVA) \\
\hline Nold & 60 & $\begin{array}{l}6.1 \\
( \pm 0.1 \mathrm{SE})\end{array}$ & $\begin{array}{l}\mathrm{F}=26.7, \mathrm{p}<0.00001 \\
(\text { Nold }>\mathrm{AD}>\mathrm{DLB})\end{array}$ & $\begin{array}{l}9.2 \\
( \pm 0.1 \mathrm{SE})\end{array}$ & $\begin{array}{l}F=39.6, p<0.00001 \\
(\text { Nold }>A D>D L B)\end{array}$ \\
\hline $\mathrm{AD}$ & 60 & $\begin{array}{l}5.5 \\
( \pm 0.1 \mathrm{SE})\end{array}$ & & $\begin{array}{l}8.6 \\
( \pm 0.1 \mathrm{SE})\end{array}$ & \\
\hline DLB & 60 & $\begin{array}{l}4.8 \\
( \pm 0.1 \mathrm{SE})\end{array}$ & & $\begin{array}{l}7.2 \\
( \pm 0.1 \mathrm{SE})\end{array}$ & \\
\hline DLB VH+ & 40 & $\begin{array}{l}4.8 \\
( \pm 0.1 \mathrm{SE})\end{array}$ & $\begin{array}{l}\mathrm{F}=16.9, \mathrm{p}<0.0001 \\
(\text { Nold }>\mathrm{AD}, \mathrm{DLBVH}-; \text { Nold }>\mathrm{AD}>\mathrm{DLBVH}+; \text { DLBVH }-=\mathrm{DLBVH}+)\end{array}$ & $\begin{array}{l}7.2 \\
( \pm 0.2 \mathrm{SE})\end{array}$ & $\begin{array}{l}\mathrm{F}=25.3, \mathrm{p}<0.0001 \\
(\text { Nold, } \mathrm{AD}>\mathrm{DLBVH}+, \mathrm{DLB} \mathrm{VH}-\text { ) }\end{array}$ \\
\hline DLB VH- & 17 & $\begin{array}{l}5.1 \\
( \pm 0.2 \mathrm{SE})\end{array}$ & & $\begin{array}{l}7.4 \\
( \pm 0.3 \mathrm{SE})\end{array}$ & \\
\hline AD (sub-group) & 25 & $\begin{array}{l}5.4 \\
( \pm 0.2 \mathrm{SE})\end{array}$ & $\begin{array}{l}\mathrm{F}=17.7, \mathrm{p}<0.00001 \\
(\text { Nold }>\mathrm{AD}>\text { DLBMMSE- } \text { DLBMMSE }+)\end{array}$ & $\begin{array}{l}8.2 \\
( \pm 0.3 \mathrm{SE})\end{array}$ & $\begin{array}{l}\mathrm{F}=25.6, \mathrm{p}<0.00001 \\
(\text { Nold }>\mathrm{AD}>\text { DLBMMSE}->\text { DLBMMSE }+)\end{array}$ \\
\hline DLB MMSE+ & 26 & $\begin{array}{l}4.7 \\
( \pm 0.1 \mathrm{SE})\end{array}$ & & $\begin{array}{l}6.9 \\
( \pm 0.2 \mathrm{SE})\end{array}$ & \\
\hline DLB MMSE- & 26 & $\begin{array}{l}4.9 \\
( \pm 0.2 \mathrm{SE})\end{array}$ & & $\begin{array}{l}7.6 \\
( \pm 0.2 \mathrm{SE})\end{array}$ & \\
\hline DLB RBD+ & 42 & $\begin{array}{l}4.9 \\
( \pm 0.1 \mathrm{SE})\end{array}$ & $\begin{array}{l}\mathrm{F}=16.2, \mathrm{p}<0.0001 \\
(\text { Nold }>\mathrm{AD}>\mathrm{DLB} \text { RBD }- \text {,DLB RBD }+ \text { ) }\end{array}$ & $\begin{array}{l}7.4 \\
( \pm 0.2 \mathrm{SE})\end{array}$ & $\begin{array}{l}F=26.1, \mathrm{p}<0.0001 \\
(\text { Nold, } A D>\text { DLB RBD+ }>\text { DLB RBD-) }\end{array}$ \\
\hline DLB RBD- & 14 & $4.8( \pm 0.3 \mathrm{SE})$ & & $6.7( \pm 0.3 \mathrm{SE})$ & \\
\hline
\end{tabular}

STATISTICAL ANOVA INTERACTION AMONG GROUP, BAND AND ROI

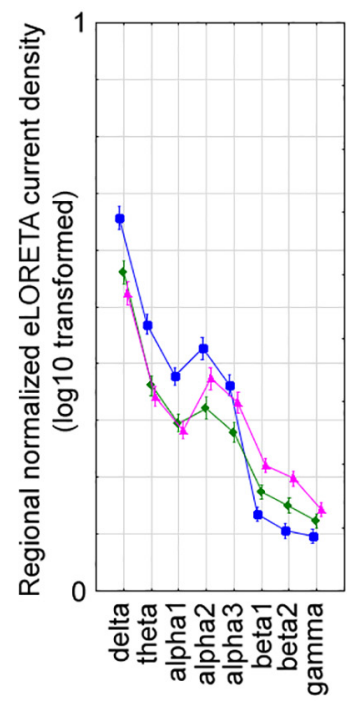

Frontal

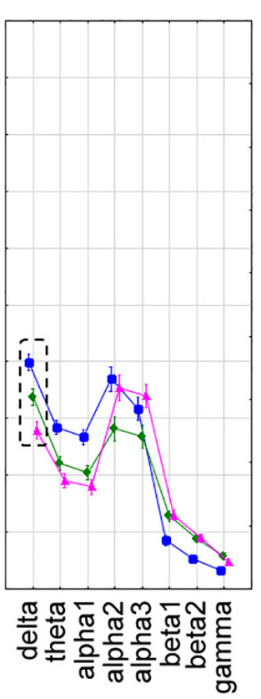

Central

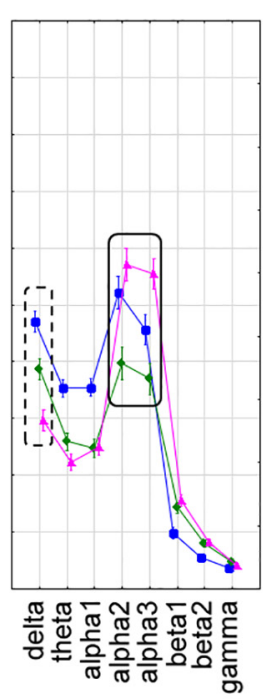

Parietal

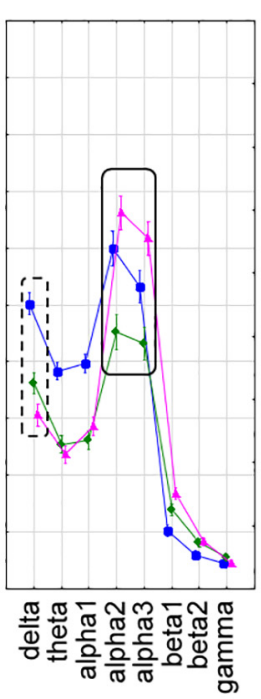

Occipital

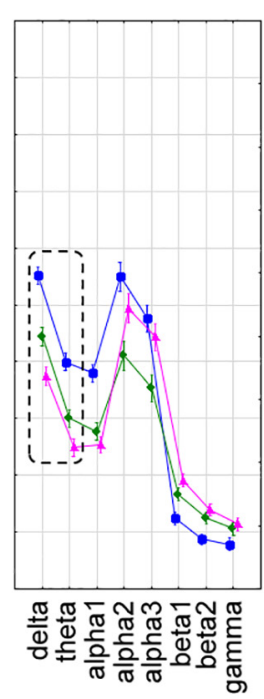

Temporal

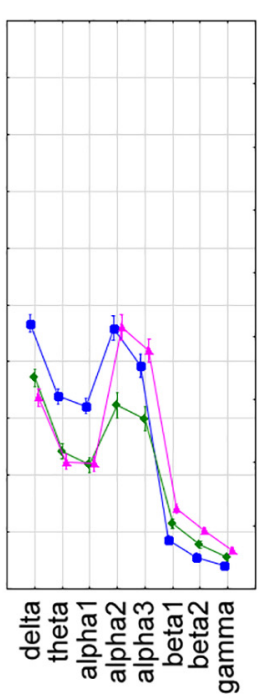

Limbic

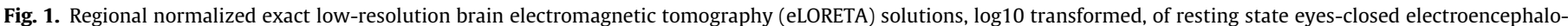

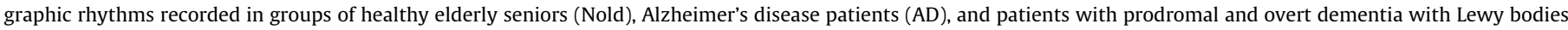

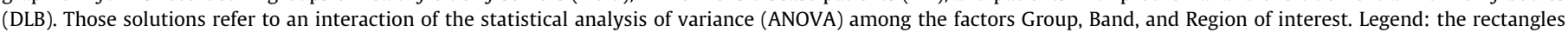
indicate the cortical regions and frequency bands in which the eLORETA solutions presented a statistically significant eLORETA pattern "Nold $\neq$ AD $\neq$ DLB" $(p<0.005)$.

formed) relative to a statistically significant ANOVA interaction effect $(\mathrm{F}=5.4 ; \mathrm{p}<0.00001)$ among the factors Group (Nold, AD, and DLB VH-, and DLB VH+), Band (delta, theta, alpha 1, alpha 2, alpha 3 , beta 1 , beta 2 , and gamma), and ROI (frontal, central, parietal, occipital, temporal, and limbic). Compared to the Nold group, the AD, DLB VH-, and DLB VH+ sub-groups showed a substantial decrease of the eLORETA solutions in posterior alpha 2 and alpha 3 sources and a substantial increase of the eLORETA solutions in widespread delta sources. The decrease of the alpha eLORETA solutions was higher in the AD than the DLB VH- and DLB VH+ subgroups, whereas the increase of the delta eLORETA solutions was higher in the DLB VH+ than the DLB VH- and AD sub-groups.

The Duncan planned post-hoc testing showed that the discriminant source pattern DLB VH+ > DLB VH- was fitted by parietal delta $(p<0.005)$. Of note, these findings were not due to outliers from those individual eLORETA solutions, as shown by Grubbs' test 


\section{STATISTICAL ANOVA INTERACTION AMONG GROUP, BAND AND ROI}

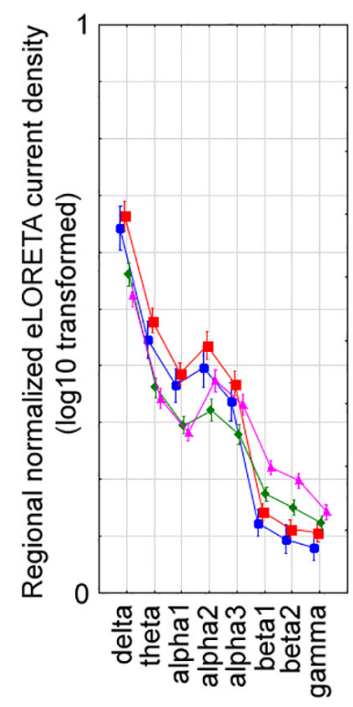

Frontal

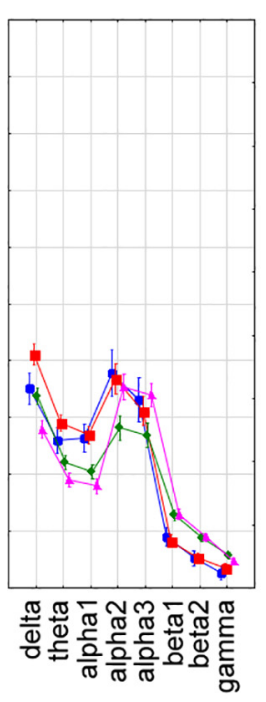

Central

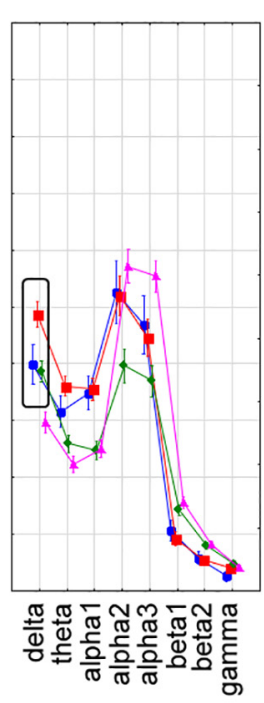

Parietal

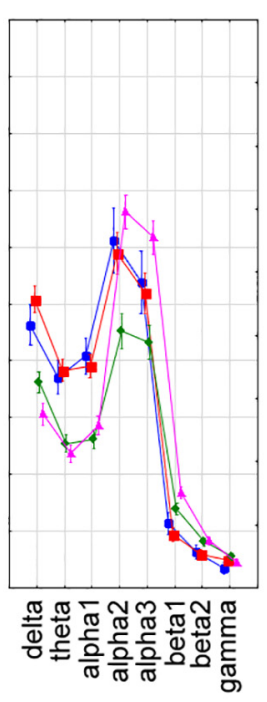

Occipital

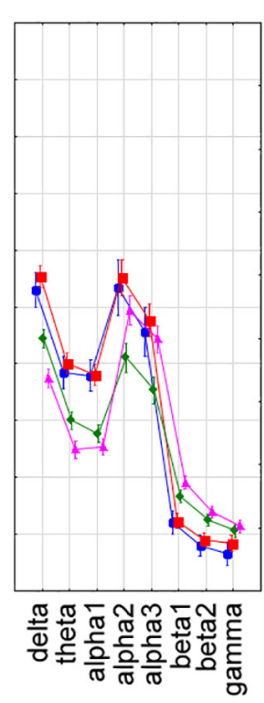

Temporal

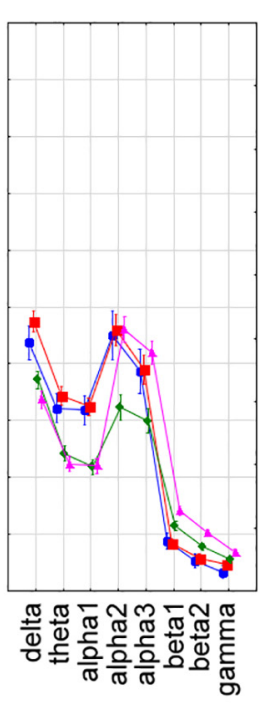

Limbic

- DLB VH

II DLB VH+

F AD Nold

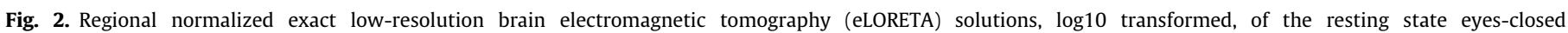

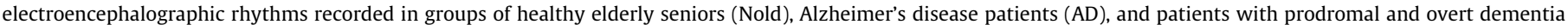

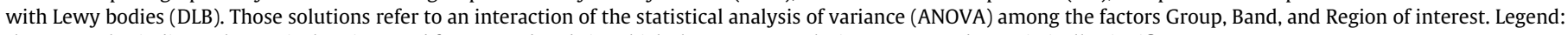

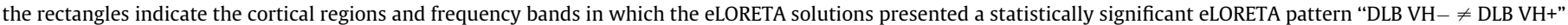
$(\mathrm{p}<0.005)$. Legend: $\mathrm{VH}=$ visual hallucinations; $\mathrm{VH}+$ and $\mathrm{VH}-=$ sub-groups of DLB patients with and without visual hallucinations, respectively.

with an arbitrary threshold of $\mathrm{p}<0.0001$ (see Fig. 2 in Supplementary Materials).

3.3. Statistical comparison of the rsEEG source activities in the Nold, $A D, D L B M M S E+$, and DLB MMSE-sub-groups

Table 2 reports the mean values of TF and IAF for the four subgroups (i.e., Nold, AD, and DLB MMSE-, and DLB MMSE+), together with the results of the statistical comparisons between the subgroups (ANOVA, $\mathrm{p}<0.05)$. The mean TF was greater $(\mathrm{F}=17.7$, $\mathrm{p}<0.00001)$ in the Nold $(\mathrm{p}<0.05)$ than in AD, DLB MMSE - and DLB MMSE+ sub-group; (2) the mean IAF was greater $(F=25.6$, $\mathrm{p}<0.00001)$ in the Nold $(\mathrm{p}<0.005)$ than AD, DLB MMSE- and DLB MMSE+ sub-groups.

Fig. 3 shows the grand average of regional rsEEG source activities (i.e., regional normalized eLORETA solutions $\log 10$ transformed) relative to a statistically significant ANOVA interaction effect $(F=5.85 ; p<0.00001)$ among the factors Group (Nold, AD, and DLB MMSE-, and DLB MMSE+), Band (delta, theta, alpha 1, alpha 2 , alpha 3 , beta 1 , beta 2 , and gamma), and ROI (frontal, central, parietal, occipital, temporal, and limbic). Compared to the Nold group, the AD, DLB MMSE-, and DLB MMSE+sub-groups showed a substantial decrease of the eLORETA solutions in posterior alpha 2 and alpha 3 sources and a substantial increase of the eLORETA solutions in widespread delta sources. The decrease of the alpha eLORETA solutions was higher in the DLB MMSE - than in DLB MMSE+ sub-group, whereas the increase of the delta eLORETA solutions was higher in the DLB MMSE+ than DLB MMSE- subgroup.

The Duncan planned post-hoc testing showed that the discriminant source pattern DLB MMSE+ > DLB MMSE- was fitted by parietal and occipital theta $(\mathrm{p}<0.01)$ as well as parietal, and occipital alpha1 ( $\mathrm{p}<0.01$ ) and central and parietal alpha2 (from $\mathrm{p}<0.005$ to $\mathrm{p}<0.01$ ). Of note, these findings were not due to outliers from those individual eLORETA solutions, as shown by Grubbs' test with an arbitrary threshold of $\mathrm{p}<0.0001$ (see Fig. 3 in Supplementary Materials).

3.4. Statistical comparison of the rSEEG source activities in the Nold, $A D$, and $D L B R B D$ - and DLB RBD+ sub-groups

Table 2 reports the mean values of TF and IAF for the four subgroups (i.e., Nold, AD, DLB RBD - and DLB RBD+), together with the results of the statistical comparisons between the sub-groups (ANOVA, $\mathrm{p}<0.05)$. The mean $\mathrm{TF}$ was greater $(\mathrm{F}=16.2$, $\mathrm{p}<0.0001)$ in the Nold and AD $(\mathrm{p}<0.005)$ than the DLB RBDand DLB RBD+ sub-groups. Furthermore, the mean IAF was greater $(F=26.1, p<0.0001)$ in the Nold and the $A D(p<0.005)$ than in DLB RBD-, and DLB RBD+ sub-groups.

Fig. 4 shows the grand average of regional rsEEG source activities (i.e., regional normalized eLORETA solutions $\log 10$ transformed) relative to a statistically significant ANOVA interaction effect $(F=5.2 ; p<0.00001)$ among the factors Group (Nold, AD, and DLB RBD-, and DLB RBD+), Band (delta, theta, alpha 1, alpha 2 , alpha 3 , beta 1 , beta 2 , and gamma), and ROI (frontal, central, parietal, occipital, temporal, and limbic). Compared to the Nold group, the AD, DLB RBD-, and DLB RBD+ sub-groups showed a substantial increase of the eLORETA solutions in widespread delta sources. Remarkably, the DLB RBD- sub-group shows an increment of posterior alpha source activity as well as occipital and temporal delta and theta sources compared to AD and DLB RBD+ sub-groups.

The Duncan planned post-hoc testing showed that the discriminant source pattern DLB RBD- > DLB RBD+ was fitted by central alpha2 ( $p<0.005$ ). Of note, these findings were not due to outliers from those individual eLORETA solutions, as shown by Grubbs' test with an arbitrary threshold of $\mathrm{p}<0.0001$ (see Fig. 4 in Supplementary Materials). 


\section{STATISTICAL ANOVA INTERACTION AMONG GROUP, BAND AND ROI}

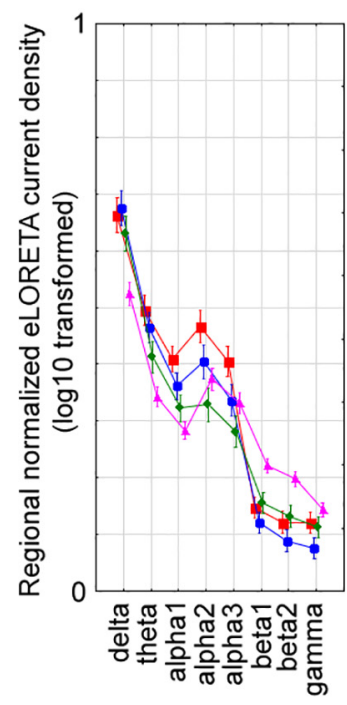

Frontal

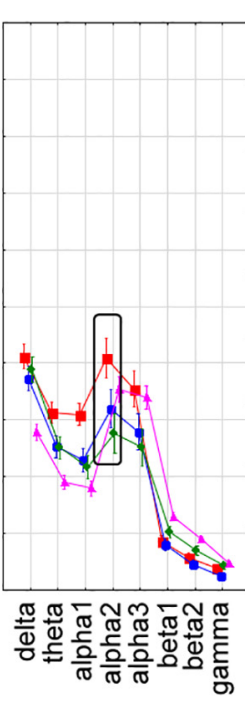

Central

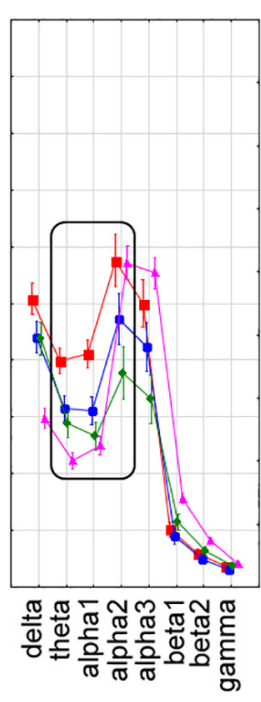

Parietal

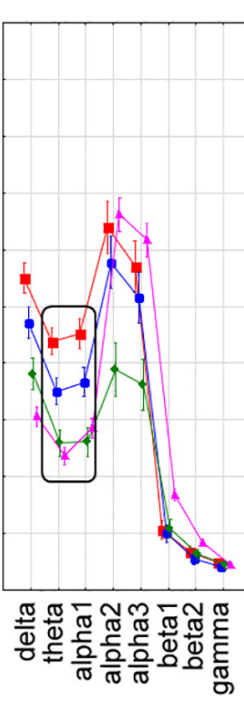

Occipital

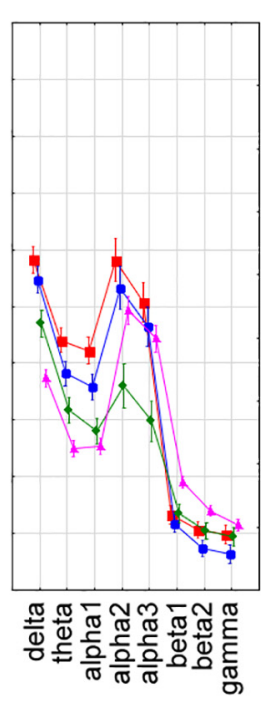

Temporal

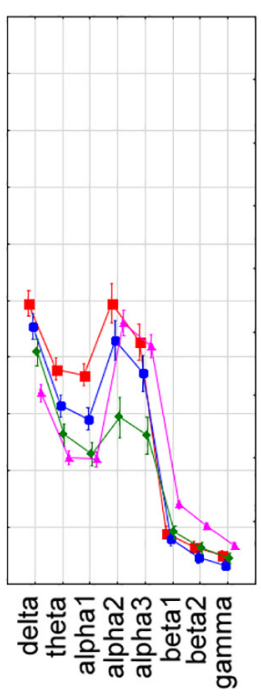

Limbic

DLB MMSE-

II DLB MMSE+

F AD Nold

DLB MMSE+ > DLB MMSE- $(p<0.01)$

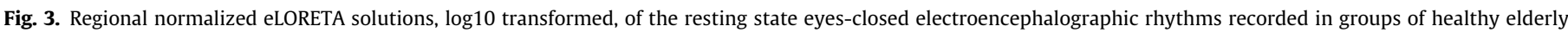

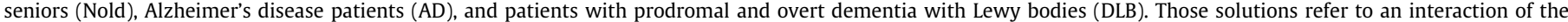

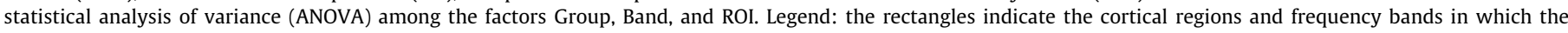

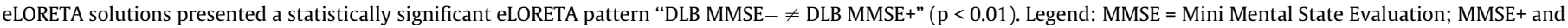
MMSE - = sub-groups of DLB patients with greater and lesser cognitive deficits, respectively.

\section{STATISTICAL ANOVA INTERACTION AMONG GROUP, BAND AND ROI}

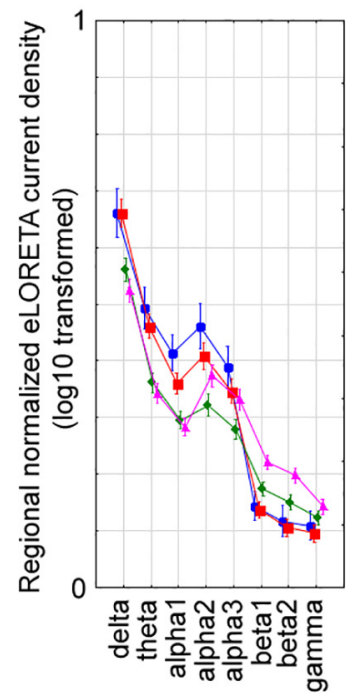

Frontal

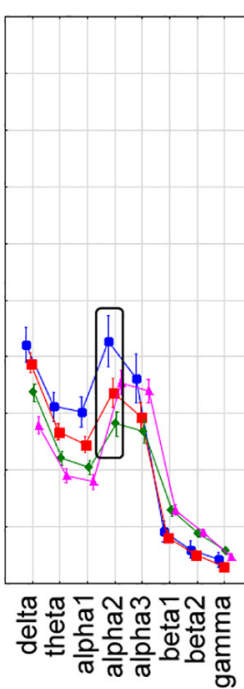

Central

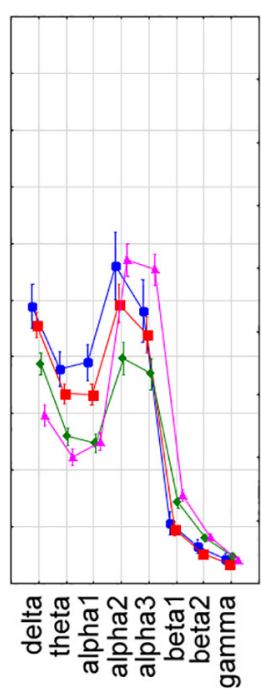

Parietal

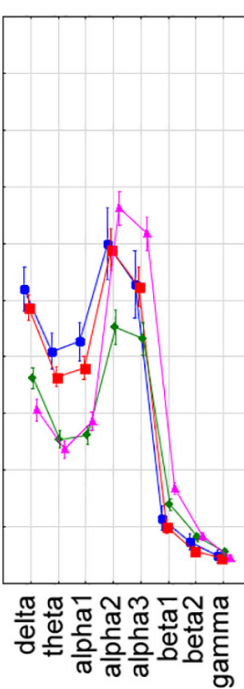

Occipital

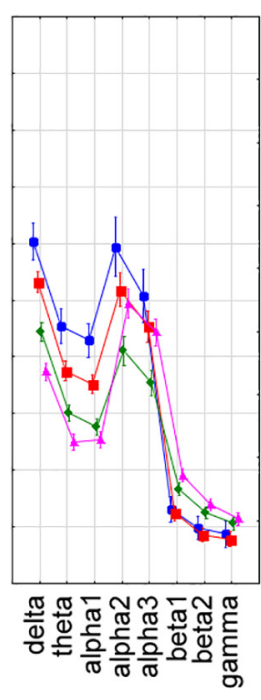

Temporal

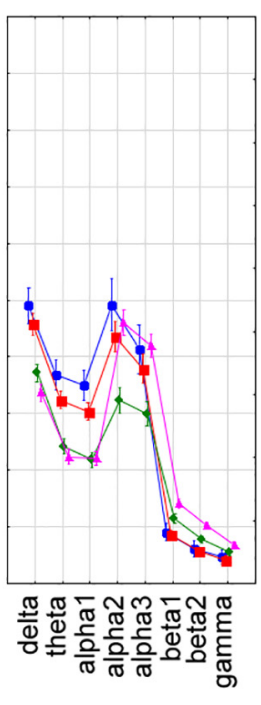

Limbic

F DLB RBD-

II DLB RBD+

F $\mathrm{AD}$ Nold

DLB RBD- > DLB RBD+ $(p<0.005)$

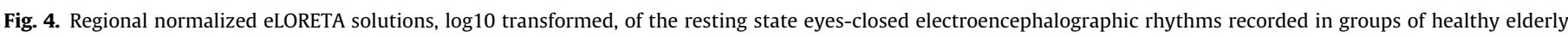

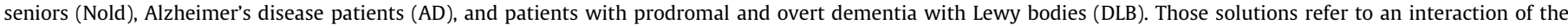

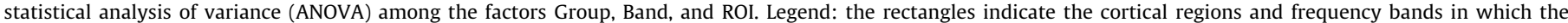

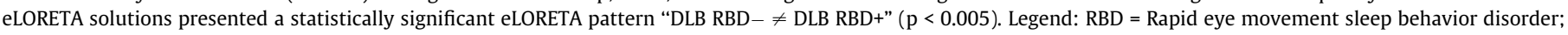
$\mathrm{RBD}+$ and $\mathrm{RBD}-{ }_{-}$sub-groups of DLB patients with and without rapid eye movement sleep behavior disorder, respectively.

\subsection{Control analysis at the group level}

Table 3 reports statistical results of the ANOVAs performed on the normalized power density of the five independent components of scalp rsEEG rhythms calculated in all DLB and Nold participants as a whole group.

Concerning the global cognitive status (i.e., MMSE score), the five ANOVAs (one for each independent component) showed a sta- 
Table 3

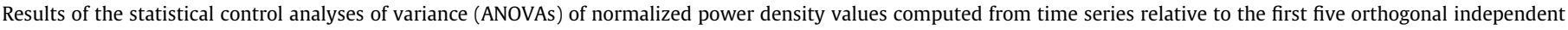

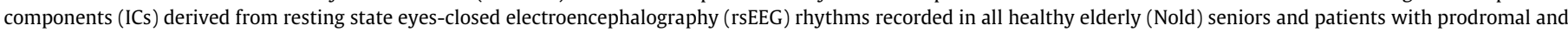
overt dementia with Lewy bodies (DLB) considered as a whole group ( $\mathrm{p}<0.01$ ).

\begin{tabular}{|c|c|c|c|}
\hline \multicolumn{4}{|c|}{$\begin{array}{l}\text { rsEEG Independent component analysis (ICA) } \\
\text { Nold vs DLB VH+ vs DLB VH- }\end{array}$} \\
\hline ICA Components & Interaction & $F$ and $p$ values & Duncan post hoc \\
\hline ICA-4 (7.1\%) & Group X Band & $\begin{array}{l}F=8.7 \\
p<0.00001\end{array}$ & $\begin{array}{l}\text { DLB VH+ }>\text { DLB VH-: } \\
\text { delta }(p<0.01)\end{array}$ \\
\hline \multicolumn{4}{|c|}{ Nold vs DLB MMSE - vs DLB MMSE+ } \\
\hline ICA Components & Interaction & $F$ and $p$ values & Duncan post hoc \\
\hline ICA-1 (55.92\%) & Group X Band & $\begin{array}{l}F=9.6 \\
p<0.00001\end{array}$ & $\begin{array}{l}\text { DLB MMSE+ > DLB MMSE-: } \\
\text { theta, alpha1, alpha2 }(\mathrm{p}<0.005)\end{array}$ \\
\hline ICA-2 (9.31\%) & Group X Band & $\begin{array}{l}\mathrm{F}=7.7 \\
\mathrm{p}<0.00001\end{array}$ & $\begin{array}{l}\text { DLB MMSE+ > DLB MMSE-: } \\
\text { theta, alpha } 1(\mathrm{p}<0.005)\end{array}$ \\
\hline$I C A-3(8.39 \%)$ & Group X Band & $\begin{array}{l}\mathrm{F}=9.0 \\
\mathrm{p}<0.00001\end{array}$ & $\begin{array}{l}\text { DLB MMSE+ > DLB MMSE-: } \\
\text { theta, alpha1, alpha2 }(\mathrm{p}<0.005)\end{array}$ \\
\hline$I C A-4(7.1 \%)$ & Group X Band & $\begin{array}{l}\mathrm{F}=10.1 \\
\mathrm{p}<0.00001\end{array}$ & $\begin{array}{l}\text { DLB MMSE+ > DLB MMSE-: } \\
\text { theta, alpha1, alpha2 }(\mathrm{p}<0.005)\end{array}$ \\
\hline$I C A-5(5.0 \%)$ & Group X Band & $\begin{array}{l}\mathrm{F}=8.6 \\
\mathrm{p}<0.00001\end{array}$ & $\begin{array}{l}\text { DLB MMSE+ > DLB MMSE-: } \\
\text { theta }(p<0.005)\end{array}$ \\
\hline \multicolumn{4}{|c|}{ Nold vs DLB RBD - vs DLB RBD+ } \\
\hline ICA Components & Interaction & $F$ and $p$ values & Duncan post hoc \\
\hline$I C A-4(7.1 \%)$ & Group X Band & $\begin{array}{l}F=7.7 \\
p<0.000001\end{array}$ & $\begin{array}{l}\text { DLB RBD }->\text { DLB RBD+ : } \\
\text { alpha2 }(\mathrm{p}<0.01)\end{array}$ \\
\hline
\end{tabular}

tistically significant interaction effect $(p<0.01)$ between the two factors Group (Nold, DLB-MMSE-, and DLB-MMSE+) and Band (from delta to gamma). The Duncan planned post-hoc testing showed the following significant differences between DLB-MMSE + and DLB-MMSE-: (1) The normalized power density of all five independent components was greater in the DLB-MMSE+ (more cognitive deficits) than the DLB-MMSE - sub-group at the theta band ( $p<0.005$ ); (2) The normalized power density of the first four independent components (i.e. from IC1 to IC4) was greater in the DLB-MMSE + than the DLB-MMSE - sub-group at the alpha 1 band ( $p<0.005$ ); and (3) The normalized power density of the independent components 1-3-4 (i.e. IC1-IC3-IC4) was greater in the DLBMMSE+ than the DLB-MMSE - sub-group at the alpha 2 band $(\mathrm{p}<0.001)$.

Concerning REM sleep behavior disorders, the five ANOVAs showed an effect only for the normalized power density of the independent component-4 (i.e. IC4), which was lower in the DLB$\mathrm{RBD}+$ (presence of sleep disorders) than the DLB-RBD- subgroup at the alpha $2(\mathrm{p}<0.01)$ band.

Concerning visual hallucinations, the five ANOVAs (one for each independent component) showed a statistically significant interaction effect between the two factors Group (Nold, DLB-VH-, and DLB-VH+) and Band (from delta to gamma) $(\mathrm{p}<0.01)$. The Duncan planned post-hoc testing $(\mathrm{p}<0.01)$ unveiled that the normalized power density of all five independent components was greater in the DLB-VH+ (presence of visual hallucination) than the DLB$\mathrm{VH}-$ sub-group at the delta band $(\mathrm{p}<0.01)$.

\subsection{Control analyses at the individual level}

The Spearman test $(\mathrm{p}<0.05$ ) evaluated the correlation between the MMSE score and rsEEG source activities showing statistically significant differences between the DLB MMSE - and DLB MMSE+ groups (i.e., occipital and parietal theta, parietal alpha 1 , and central and parietal alpha 2 source activities). A statistically significant negative correlation was found between the MMSE score vs. parietal and occipital theta and alpha1 eLORETA solutions in all DLB subjects $(\mathrm{r}=-0.39$ to $-0.33, \mathrm{p}<0.05)$. The higher delta and theta source activities, the lower the MMSE score (the greater the global cognitive deficits), see Table 4.
The rsEEG source activities showing statistically significant differences between the DLB MMSE- and DLB MMSE+ groups were also used as an input to the PLS regression on the MMSE score. The PCA extracted 2 principal components (latent variables) explaining $7.7 \%$ of the sum of squares in the dependent variables. Only the first principal component was statistically significant ( $p<0.05$ ), explaining $79.2 \%$ of the variability in the predictors and $6.2 \%$ of the variability in the dependent variable (i.e., the "response"). Furthermore, the best predictor was the occipital theta source activity, with a VIP of 0.47 (Spearman's test, $\mathrm{p}<0.05$ ).

To compute the classification accuracy of rsEEG source activities in DLB patients, the discriminant variables were those showing statistically significant differences $(\mathrm{p}<0.01)$ between the DLB MMSE+ and DLB MMSE - sub-groups and between the DLB VH+ vs DLB $\mathrm{VH}$ - sub-groups. Indeed, no rsEEG source activity showed significant differences between the DLB RBD+ vs DLB RBD- sub-groups $(\mathrm{p}>0.01)$.

For the comparison between the DLB MMSE+ vs DLB MMSEsub-groups, the significant discriminant values were those of the central alpha 2, parietal theta, parietal alpha 1, parietal alpha 2, occipital theta, and occipital alpha 1 source activities $(p>0.01$ ). The values of these variables were averaged as a theta-alpha composite score used as an input to the ROC curve analysis.

Table 4

Results of the correlation analysis (Spearman test, $\mathrm{p}<0.05$ ) performed between the MMSE score and cortical sources of resting state eyes-closed electroencephalography (rsEEG) rhythms at delta, theta, and alpha 1 bands in DLB MMSE - and DLB MMSE+ patients considered as a whole group. In particular, these results include Spearman's correlation coefficient (R) and the associated level of significance (p). Legend: DLB $=$ prodromal and overt dementia with Lewy bodies; MMSE $=$ Mini Mental State Evaluation; MMSE+ and MMSE - = sub-groups of DLB patients with greater and lesser cognitive deficits, respectively.

$\begin{aligned} & \text { Correlation between (eLORETA) source activity of the rsEEG rhythms and } \\
& \text { MMSE score }\end{aligned}$
\begin{tabular}{lll} 
& Spearman R & $\mathrm{p}$ \\
\hline Parietal theta & $-0,33$ & 0,017 \\
Occipital theta & $-0,39$ & 0,005 \\
Parietal alpha1 & $-0,36$ & 0,009 \\
Occipital alpha1 & $-0,38$ & 0,005 \\
\hline
\end{tabular}


Table 5

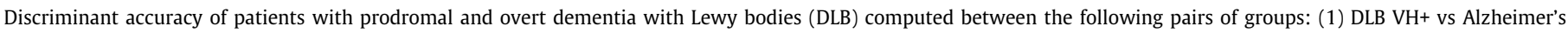

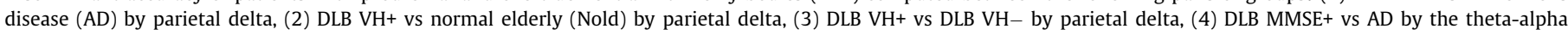

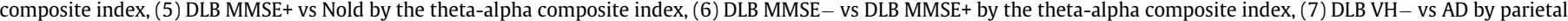

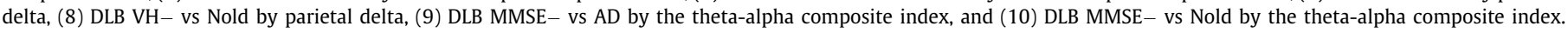

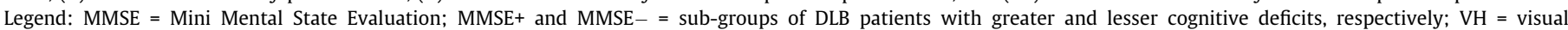
hallucinations; $\mathrm{VH}+$ and $\mathrm{VH}-=$ sub-groups of DLB patients with and without visual hallucinations, respectively.

\begin{tabular}{|c|c|c|c|c|}
\hline & Sensitivity\% & Specificity\% & Accuracy\% & AUROC curve \\
\hline DLB VH+ vs AD by parietal delta & 75 & 65 & 69 & 0.69 \\
\hline DLB VH+ vs Nold by parietal delta & 75 & 85 & 81 & 0.85 \\
\hline DLB VH+ vs DLB VH- by parietal delta & 70 & 70.6 & 70.2 & 0.69 \\
\hline DLB MMSE+ vs AD by theta-alpha composite score & 76.7 & 68 & 72.7 & 0.74 \\
\hline DLB MMSE+ vs Nold by theta-alpha composite score & 76.7 & 61.7 & 66.7 & 0.70 \\
\hline DLB MMSE - vs DLB MMSE+ by theta-alpha composite score & 76.7 & 50 & 63.3 & 0.61 \\
\hline DLB VH - vs AD by parietal delta & 70.6 & 56.7 & 59.7 & 0.58 \\
\hline DLB VH- vs Nold by parietal delta & 94.1 & 56.7 & 64.9 & 0.77 \\
\hline DLB MMSE - vs AD by theta-alpha composite score & 66.7 & 68 & 67.3 & 0.68 \\
\hline DLB MMSE - vs Nold by theta-alpha composite score & 93.3 & 38.3 & 56.7 & 0.62 \\
\hline
\end{tabular}

For the comparison between DLB VH+ vs DLB VH-groups, the significant discriminant values were those of the parietal delta source activities ( $p>0.01$ ), used as an input to the ROC analysis.

Overall, the discriminant accuracy was computed between individuals of the following pairs of groups: (1) DLB VH+ vs AD by parietal delta, (2) DLB VH+ vs Nold by parietal delta, (3) DLB VH+ vs DLB VH- by parietal delta, (4) DLB MMSE+ vs AD by the thetaalpha composite index, (5) DLB MMSE+ vs Nold by the thetaalpha composite index, (6) DLB MMSE - vS DLB MMSE+ by the theta-alpha composite index, (7) DLB VH- vs AD by parietal delta, (8) DLB VH - vs Nold by parietal delta, (9) DLB MMSE - vs AD by the theta-alpha composite index, and (10) DLB MMSE- vs Nold by the theta-alpha composite index.

Results showed that the best discriminant accuracies (AUROC curve) were reached in the discrimination between the DLB VH+ vs. Nold seniors by parietal delta source activity (0.85), the DLB VH - vs. Nold seniors by parietal delta source activity (0.77), the DLB MMSE+ vs. AD seniors by the theta-alpha composite score (0.74), and the DLB MMSE+ vs. Nold seniors by the theta-alpha composite score (0.70). All results were reported in Table 5.

\section{Discussion}

In the present retrospective and exploratory rsEEG study, we hypothesized that the topography of cortical delta and alpha source activities may differ in sub-groups of demographically matched DLB patients as a function of characterizing disease symptoms such as cognitive deficits, REM sleep behavior disorders, and visual hallucinations. The exploratory nature was reflected by liberal statistical thresholds $(\mathrm{p}<0.05-0.01)$ that might produce potential false positive discoveries. Overall, the present study aimed to provide preliminary evidence that some rsEEG source activities may be related to relevant clinical features in DLB patients and may be considered as candidate neurophysiological markers of those features to be cross validated in future prospective and longitudinal studies. Specifically, these neurophysiological markers may probe oscillatory neural synchronization mechanisms in brainstem-cortical, forebrain-cortical, and thalamuscortical circuits regulating the brain arousal and vigilance in quiet wakefulness, as one of the neurophysiological bases of higher functions in humans.

\subsection{Increase in delta and reduction in alpha rsEEG source activities in DLB patients}

In the present study, an initial analysis of rsEEG rhythms contrasted delta and alpha source activities in the whole DLB group compared with the control Nold and AD subjects. In relation to the Nold group, the AD and DLB groups were characterized by greater delta source activities in widespread cortical regions and lower alpha source activities in posterior cortical lobes. The abnormalities in delta source activities were greater in the DLB than the AD group, while those in alpha source activities were prominent in the $\mathrm{AD}$ than the $\mathrm{DLB}$ group. Overall, these findings replicated previous rsEEG evidence observed in AD and DLB patients (Bonanni et al., 2008, 2015; Babiloni et al., 2017a,b).

According to the neurophysiological animal model by Cardiff group (Hughes and Crunelli, 2005; and Lörincz et al., 2008, 2009), the present abnormalities in cortical alpha source activities might reflect an alteration in the interplay of cholinergic projections from basal forebrain to the following oscillating neural networks involving glutamatergic high-threshold and relay-mode thalamocortical neurons, GABA interneurons, and cortical pyramidal neurons. From a translational point of view, a recent rsEEG study has reported abnormal alpha source activities in restingstate brain neural networks in DLB patients (Aoki et al., 2019). In that study, occipital alpha source activity was reduced in DLB patients over healthy controls in a visual neural network, this abnormality being correlated with deficits in attention, visuospatial skills, and cognition possibly reflecting cholinergic impairment (Aoki et al., 2019).

Concerning the present abnormalities in delta source activities observed in AD and DLB patients, it may be generated by an abnormal synchronization of neurons of thalamus and cerebral cortex, possibly related to an altered functional connectivity in parietal, temporal, and occipital regions (Steriade \& Llinás, 1988; Pfurtscheller and Lopes da Silva, 1999).

\subsection{Increase in delta and alpha source activities in DLB patients with marked cognitive deficits and visual hallucinations}

As novel findings of the present study, the DLB patients with greater cognitive deficits over the DLB controls exhibited higher alpha source activity in posterior regions (e.g., central, occipital, and parietal). Furthermore, the DLB patients with visual hallucinations over the DLB controls displayed higher parietal delta source activities. It should be remarked that the above differences in delta and alpha source activities were unrelated to basic confounding variables in the various statistical contrasts (i.e., global motor and cognitive deficits, visual hallucinations, REM behavioral disorders, disease duration, and psychoactive therapies with dopaminergic, anti-psychotic and cholinergic).

Concerning the above abnormalities in delta source activities, the present results complement previous rsEEG findings derived from the comparison of scalp rsEEG markers between DLB patients 
and subjects belonging to Nold, DLB, PDD, and ADD groups (Walker et al., 2000a,b; Kai et al., 2005; Andersson et al., 2008; Bonanni et al., 2008; Babiloni et al., 2017a). Summarizing, those previous findings showed the following effects. Compared with Nold and ADD groups, DLB patients exhibited greater delta and theta rhythms (Andersson et al., 2008; Kai et al., 2005; Stylianou et al., 2018). Previous findings showed that DLB patients with hallucinations have greater widespread delta rhythms when compared to ADD patients with hallucinations (Dauwan et al., 2018). In this framework, the present results demonstrated for the first time that DLB patients with greater cognitive deficits, and visual hallucinations can be distinguished by different spatial patterns of delta source activities. Therefore, future studies may test the association of this different symptoms-related delta source topography with DLB neuropathological processes such as cortical $\alpha$ synucleinopathy and intracellular Lewy bodies (Caviness et al., 2018) as well as total phosphorylated $\boldsymbol{\alpha}$-synuclein (Caviness et al., 2016), beyond the concept of "global delta rhythms" in quiet wakefulness.

Concerning the above abnormalities in alpha source activities, the present results apparently challenge previous rsEEG evidence derived from the comparison of rsEEG markers among Nold, DLB, PDD, and ADD groups (Babiloni et al., 2017a; Dauwan et al., 2018; Aoki et al., 2019). Such previous rsEEG evidence pointed to lower posterior alpha source activities in DLB patients compared with Nold subjects (Babiloni et al., 2017a; Aoki et al., 2019). It also showed higher frontal-parietal functional connectivity at scalp alpha rhythms in DLB patients with hallucinations compared with ADD patients with similar hallucinations (Dauwan et al., 2018). At the present early stage of the research, we can just speculate about this challenge, namely the present result of greater posterior alpha source activities in DLB patients with visual hallucinations over the DLB controls. Our speculation is grounded on the following results of our recent rsEEG studies in PD patients with cognitive deficits. In one study, we reported that (1) an acute dose of levodopa induced a decrease in both widespread delta and alpha source activities in these PD patients and (2) alpha source activities were greater in the PD patients with major cognitive deficits over the PD controls (Babiloni et al., 2019). In another study, we reported that the PD patients with visual hallucinations over the PD controls had greater alpha source activities, while those with greater motor deficits were characterized by lower posterior alpha source activities with no effects on delta source activities (Babiloni et al., 2020b). These previous results suggest that PD processes might affect the activity of parallel dopaminergic sub-systems exerting opposite effects on the thalamus-cortical generation of alpha rhythms in quiet wakefulness. On one hand, the PD-related decrease in nigrostriatal dopaminergic neurotransmissions might induce a marked reduction in posterior alpha source activities related to motor deficits, possibly reflecting a background tonic increase in resting state cortical arousal. On the other hand, the PD-related decrease in mesolimbic and mesocortical dopaminergic neurotransmissions might induce a slight increase in posterior alpha source activities related to cognitive deficits and visual hallucinations. This reduction might be related to a tonic decrease in the cortical arousal. Here we extend this speculation to the present DLB patients with visual hallucinations. These patients might suffer from prevalent alterations in mesolimbic and mesocortical dopaminergic neurotransmissions inducing a background tonic decrease in the cortical arousal during the resting state condition and relatively higher posterior alpha source activities estimated from rsEEG rhythms compared with the DLB controls. This effect might be hidden in the comparison of rsEEG markers between DLB patients and the control groups including Nold subjects or patients with ADD and PDD, thus emphasizing the importance of statistical comparisons of rsEEG markers not only between DLB and other groups of Nold,
$A D$, and PD patients but also between matched DLB sub-groups with characteristic clinical features. Of course, the present alpha source abnormalities in DLB sub-groups might not be only due to dysfunctions in the dopaminergic systems and might be associated with alterations in ascending activating systems using other neurotransmitters (e.g., noradrenergic, cholinergic, etc.). More research is needed before any final conclusions.

\subsection{Decrease in alpha source activities in DLB patients with REM sleep behavior disorders}

With the same methodological approach, here we contrasted delta and alpha source activities in matched DLB sub-groups without vs. with REM sleep behavior disorders (i.e., parasomnia with vivid, often frightening dreams related to motor behaviors during REM sleep). The DLB patients with REM sleep behavior disorders over the DLB controls were characterized by lower alpha source activities in central cortical region. This effect was unrelated to the mentioned confounding variables (global cognitive and motor deficits, visual hallucinations, etc.) and complements previous evidence indicating abnormal spectral EEG features (i.e. frequency slowing of rsEEG rhythms) and poor muscular atonia in DLB patients examined during REM sleep (Fantini et al., 2003; Massicotte-Marquez et al., 2005; Iranzo et al., 2010; Inoue et al., 2015).

Unfortunately, we have no conclusive explanation why the present DLB patients with REM sleep behavior disorders. Indeed, this effect is apparently in disagreement with the increase in delta rhythms observed in previous rsEEG studies carried out in patients with DLB compared with Nold and ADD subjects (Walker et al., 2000a,b; Kai et al., 2005; Andersson et al., 2008; Bonanni et al., 2008; Babiloni et al., 2017a). Furthermore, it is partially in disagreement with previous rsEEG findings showing that MCI patients with REM sleep behavior disorders have a higher parietal, temporal and occipital theta rhythms, and lower occipital alpha rhythms, when compared to subjects with REM sleep behavior disorders but no cognitive deficits (Rodrigues Brazete et al., 2013). In a previous rsEEG study, subjects with REM sleep behavior disorders pointed to greater frontal, temporal, and occipital theta rhythms and lower alpha rhythms in relation to Nold subjects (Fantini et al., 2003; Iranzo et al., 2010).

As a tentative explanation of the present findings, we still refer to the mentioned results of our recent rsEEG studies showing that PD patients with major cognitive deficits over PD controls had greater delta and alpha source activities, while an acute dose of levodopa decreased both widespread delta and alpha source activities (Babiloni et al., 2019). Keeping in mind the tentative explanation given in the previous section on cognitive deficits and visual hallucinations, we speculate that the present DLB patients with REM sleep behavior disorders might suffer from a major impairment in nigrostriatal dopaminergic neurotransmissions inducing a background tonic increase in the resting state cortical arousal, reflected by a desynchronization in alpha rhythms. Such impairment might involve brainstem neural circuits using several neurotransmitters. See the basis of this speculation in the following paragraphs.

In Lewy body diseases (e.g., PDD, DLB), poor muscle atonia and dream enactment behavior during REM sleep might be partially due to poor noradrenergic projections that origin from locus coeruleus and do target amygdala, thalamus, and cerebral cortex (Jones et al., 1977; Cash et al., 1987; Zweig et al., 1993; Del Tredici and Braak, 2013). Those projections also target raphe (serotoninergic) and substantia nigra (dopaminergic) nuclei of ascending activating systems regulating the brain arousal and possibly rsEEG rhythms (Vermeiren and De Deyn, 2017). As a matter of fact, DLB patients are characterized by significant neural losses in the locus coeruleus 
due to $\alpha$-synuclein pathology, although the relation with REM sleep behavior disorders is still under discussion (Dugger et al., 2012; Del Tredici and Braak, 2013). Furthermore, lesions in the cat locus coeruleus interfered with muscle atonia and pontogeniculo-occipital waves in REM sleep stages (Jones et al., 1977).

In the same line of speculation, REM sleep behavior disorders might be also related to poor cholinergic projections from pedunculopontine tegmental nucleus (PPT) and Meynert nucleus of cholinergic basal forebrain. As a matter of fact, DLB patients with REM sleep behavior disorders suffer from significant neural loss in PPT due to $\alpha$-synuclein pathology (Del Tredici and Braak, 2013). Furthermore, DLB patients treated with acetylcholinesterase inhibitors showed a mitigation of REM sleep behavior disorders (Massironi et al., 2003). Finally, recent optogenetic evidence in mice hints that both PPT and cholinergic basal forebrain are directly (and diversely) involved in the modulation of brain arousal to enhance information processing of salient stimuli and behavioral reactions (Azzopardi et al., 2018; Aitta-Aho et al., 2018). In this speculative line, ascending pedunculopontine cholinergic projections to midbrain dopaminergic nuclei (i.e., substantia nigra and ventral tegmental area) might modulate rewardseeking and behavior (Yeomans, 2012), while parallel descending cholinergic pedunculopontine projections to pons reticular formation might modulate thalamocortical activity at alpha rhythms regulating cortical arousal in visual systems and relative functions such as vigilance and visual (hypnagogic) imagery. The alteration of these functions in DLB patients might be one of the neural bases of visual hallucinations and abnormalities in dreams and REM sleep (Yeomans, 2012). Finally, Meynert nucleus of cholinergic basal forebrain may enhance sensory information processing in the cerebral cortex via the regulation of parieto-occipital alpha rhythms in wakefulness (Bentley et al., 2008; Janzen et al., 2012).

\subsection{Methodological remarks}

In this exploratory study, the interpretation of the results should be done considering some significant methodological limitations.

In seniors with cognitive deficits, the analysis of posterior dominant rsEEG rhythms should consider not only rsEEG waveform shapes (e.g., EEG epileptiform activities, intermittent or persistent EEG slow-frequency oscillations, etc.), topography, and power peak frequency but also the reduction in amplitude of those rhythms possibly due to eyes opening, drowsiness, sleep onset, and/or brain neuropathology (Babiloni et al., 2020a). In the present retrospective and exploratory study, we carefully controlled the presence of behavioral and EEG signs of drowsiness or sleep onset in the preliminary data analysis phase (see Methods). Furthermore, we took into account the typical slowing in frequency of posterior dominant alpha rhythms in pathological aging using individual frequency bands anchored on the IAF peak (Klimesch, 1999). However, a fine distinction between posterior alpha rhythms slowed in power peak frequency vs. an abnormal increase in posterior theta rhythms could not be systematically corroborated by the analysis of the reactivity in the posterior dominant rhythms during eyes opening or the association with structural and/or metabolic impairments in cortical (i.e. parietal, temporal, and occipital areas) and/or thalamic nuclei. Unfortunately, the available database for the present study did not have those control data in the majority of DLB patients (Fig. 5 shows an example of the alpha power reactivity during eyes opening in a DLB patient of the present study). Therefore, the present findings should be considered as preliminary. Future multimodal prospective studies in DLB patients should include rsEEG recordings in both eye-open and -closed conditions, structural MRIs, and metabolic FDG-PET mapping to confirm those findings.

The traditional clinical 10-20 montage with 19 scalp exploring electrodes (e.g., 10-20 system) used in the present study is not ideal for an optimal spatial sampling of rsEEG rhythms aimed at estimating underlying cortical sources (Liu et al., 2018; Marino et al., 2016). Literature evidence shows that EEG source estimates using few scalp exploring electrodes may lead to both blurring of source estimates and incorrect punctual localizations (Michel and Koening, 2018), and that this estimation problem may be fatal in the localization of sources of epileptiform activity from less than 32 scalp exploring electrodes (Michel et al., 2004). Furthermore, the present study used the standard eLORETA template brain model (i.e., MNI152 developed by Montreal Neurologic Institute) rather than models derived from individual MRI scans taking into account of inter-subject variability in the shape of cortical gyri

\section{Resting-state EEG signal and spectral power density in eyes open and closed on Pz channel}
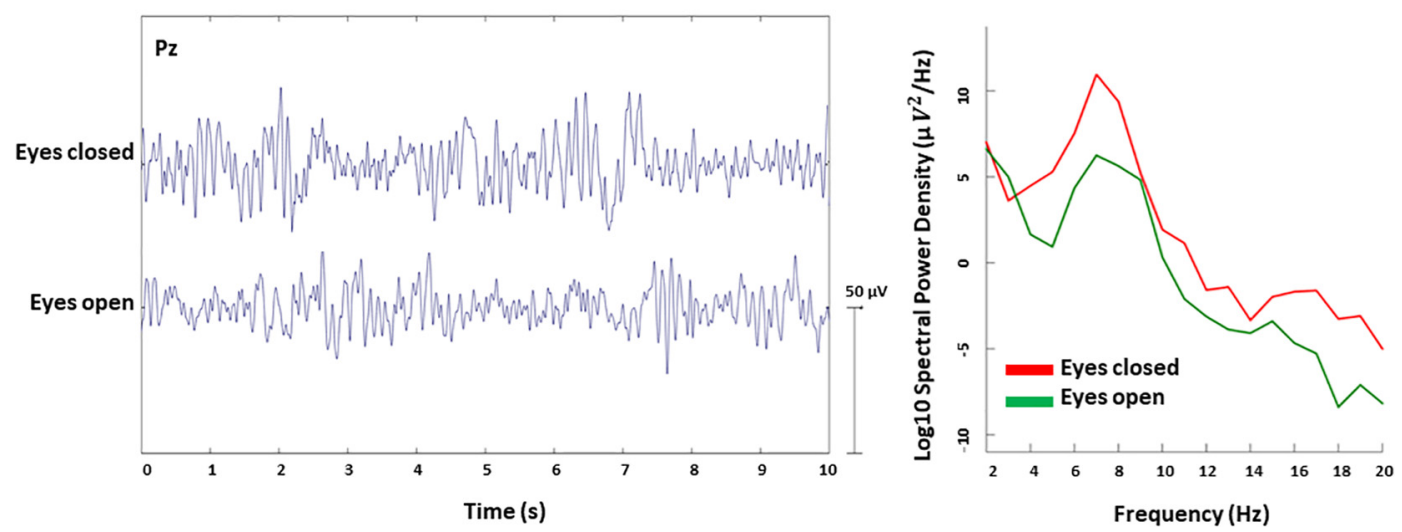

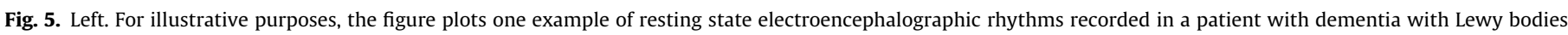

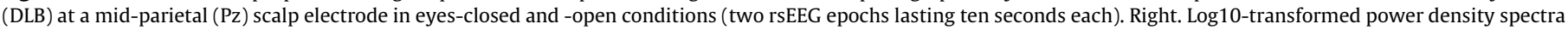

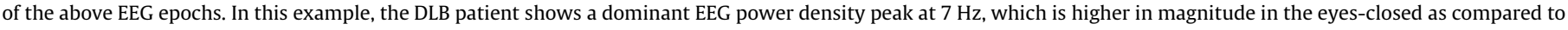

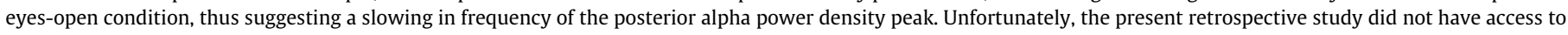

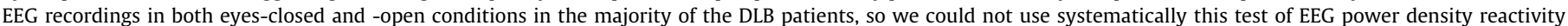

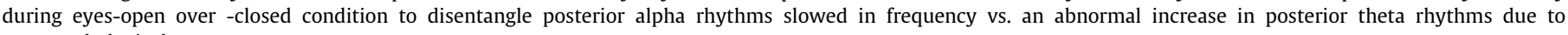
neuropathological reasons. 
and convolutions. To mitigate these methodological limitations, we averaged eLORETA solutions within broad ROIs representing cortical lobes rather than focusing on a punctual voxel-based analysis and source localization. The present source estimation at the regional level is supposed to approximate the widespread generation of rsEEG cortical rhythms occurring in quiet wakefulness at eyes closed (Babiloni et al., 2020a), as eLORETA toolbox is especially suitable to model spatially widespread source activations due to its smoothing regulation procedures (Halder et al., 2019).

These widespread source activations are expected based on spatial features of rsEEG activity (Lehmann et al., 1987; Michel and Koenig, 2018; Samogin et al., 2019; Jia \& Yu, 2019).

\section{Conclusions}

In the present retrospective and exploratory study, we tested the hypothesis that cortical sources of resting state delta and alpha rhythms may differ as a function of different clinical symptoms in matched sub-groups of DLB patients. Results showed that as compared to AD patients, DLB patients exhibited greater spatially distributed delta source activities and lower alpha source activities posteriorly, while the comparisons between matched DLB subgroups unveiled a different spatial pattern of delta and alpha source activities as a function of clinical manifestations. Specifically, (1) the DLB sub-group with the greatest REM sleep behavior disorders showed lower alpha source activities in the central cortical region; (2) those with the greatest cognitive deficits mainly showed higher theta and alpha source activities posteriorly; and (3) those with visual hallucinations pointed to greater parietal delta source activity. These results suggest that cortical neural synchronization mechanisms generating peculiar topographical abnormalities in delta and alpha source activities in quiet wakefulness are related to relevant DLB patients' clinical features at the group level. These preliminary findings encourage future crossvalidation studies in DLB patients using a significantly higher number of scalp exploring electrodes $(>48-64)$ and head models derived from individual MRI scans to estimate rsEEG source activities. Ideally, they should test the hypothesis that those neural synchronization mechanisms are directly affected by parallel DLB neuropathological processes and may explain at least in part different clinical manifestations and trajectories in DLB patients.

\section{Declaration of Competing Interest}

The authors declare that they have no known competing financial interests or personal relationships that could have appeared to influence the work reported in this paper.

\section{Acknowledgements}

The present study was developed based on the data of the informal European Consortium PDWAVES and European Consortium of Dementia with Lewy Bodies. The members and institutional affiliations of the Consortia are reported in the cover page of this manuscript. In this study, the electroencephalographic data analysis was partially supported by the funds of "Ricerca Corrente" attributed by Italian Ministry of Health to the Istituto di Ricerca e Cura a Carattere Scientifico (IRCCS) SDN of Naples, the IRCCS OASI of Troina, and the IRCCS San Raffaele Pisana of Rome. The research activities of the Unit of Sapienza University of Rome were partially supported by the H2020 Marie S. Curie ITN-ETN project with the short title "BBDiag" (http://bbdiag-itn-etn.eu) and the H2020-TWINN-2015 project with the short title "SynaNet" (https:// www.synanet2020.com).

\section{Appendix A. Supplementary material}

Supplementary data to this article can be found online at https://doi.org/10.1016/j.clinph.2020.09.004.

\section{References}

Aitta-Aho T, Hay YA, Phillips BU, Saksida LM, Bussey TJ, Paulsen O, Apergis-Schoute J. Basal forebrain and brainstem cholinergic neurons differentially impact amygdala circuits and learning-related behavior. Curr Biol 2018;28 (16):2557-2569.e4.

Andersson M, Hansson O, Minthon L, Rosén I, Londos E. Electroencephalogram variability in dementia with lewy bodies, Alzheimer's disease and controls. Dement Geriatr Cogn Disord 2008;26(3):284-90.

Aoki Y, Kazui H, Pascal-Marqui RD, Ishii R, Yoshiyama K, Kanemoto H, et al. EEG resting-state networks in dementia with lewy bodies associated with clinical symptoms. Neuropsychobiology 2019;77(4):206-18.

Azzopardi E, Louttit AG, DeOliveira C, Laviolette SR, Schmid S. The role of cholinergic midbrain neurons in startle and prepulse inhibition. J Neurosci 2018;38 (41):8798-808.

Babiloni C, Barry RJ, Başar E, Blinowska KJ, Cichocki A, Drinkenburg WHIM, et al. International Federation of Clinical Neurophysiology (IFCN) - EEG research workgroup: recommendations on frequency and topographic analysis of resting state EEG rhythms. Part 1: Applications in clinical research studies. Clin Neurophysiol 2020;131(1):285-307.

Babiloni C, Del Percio C, Lizio R, Noce G, Cordone S, Lopez S, et al. Abnormalities of cortical neural synchronization mechanisms in subjects with mild cognitive impairment due to Alzheimer's and Parkinson's Diseases: an EEG study. J Alzheimers Dis 2017a;59(1):339-58.

Babiloni C, Del Percio C, Lizio R, Noce G, Cordone S, Lopez S, et al. Abnormalities of cortical neural synchronization mechanisms in patients with dementia due to Alzheimer's and Lewy body diseases: an EEG study. Neurobiol Aging 2017b;55:143-58.

Babiloni C, Del Percio C, Lizio R, Noce G, Lopez S, Soricelli A, et al. Functional cortical source connectivity of resting state electroencephalographic alpha rhythms shows similar abnormalities in patients with mild cognitive impairment due to Alzheimer's and Parkinson's diseases. Clin Neurophysiol 2018a;129(4):766-82.

Babiloni C, Del Percio C, Lizio R, Noce G, Lopez S, Soricelli A, et al. Abnormalities of resting-state functional cortical connectivity in patients with dementia due to Alzheimer's and Lewy body diseases: an EEG study. Neurobiol Aging 2018b;65:18-40.

Babiloni C, Del Percio C, Lizio R, Noce G, Lopez S, Soricelli A, et al. Abnormalities of resting state cortical EEG rhythms in subjects with mild cognitive impairment due to Alzheimer's and Lewy body diseases. J Alzheimers Dis 2018c;62 (1):247-68.

Babiloni C, Del Percio C, Lizio R, Noce G, Lopez S, Soricelli A, et al. Levodopa may affect cortical excitability in Parkinson's disease patients with cognitive deficits as revealed by reduced activity of cortical sources of resting state electroencephalographic rhythms. Neurobiol Aging 2019;73:9-20.

Babiloni C, Pascarelli MT, Lizio R, Noce G, Lopez S, Rizzo M, et al. Abnormal cortical neural synchronization mechanisms in quiet wakefulness are related to motor deficits, cognitive symptoms, and visual hallucinations in Parkinson's disease patients: an electroencephalographic study. Neurobiol Aging 2020.

Bentley P, Driver J, Dolan RJ. Cholinesterase inhibition modulates visual and attentional brain responses in Alzheimer's disease and health. Brain 2008;131:409-24.

Bodenmann S, Rusterholz T, Dürr R, Stoll C, Bachmann V, Geissler E, et al. The functional Val158Met polymorphism of COMT predicts interindividual differences in brain alpha oscillations in young men. J Neurosci 2009;29 (35):10855-62.

Bonanni L, Perfetti B, Bifolchetti S, Taylor JP, Franciotti R, Parnetti L, et al Quantitative electroencephalogram utility in predicting conversion of mild cognitive impairment to dementia with Lewy bodies. Neurobiol Aging 2015;36 (1):434-45.

Bonanni L, Thomas A, Tiraboschi P, Perfetti B, Varanese S, Onofrj M. EEG comparisons in early Alzheimer's disease, dementia with Lewy bodies and Parkinson's disease with dementia patients with a 2-year follow-up. Brain 2008;131:690-705.

Bosboom JL, Stoffers D, Stam CJ, van Dijk BW, Verbunt J, Berendse HW, et al. Resting state oscillatory brain dynamics in Parkinson's disease: an MEG study. Clin Neurophysiol 2006;117:2521-31.

Bosboom JL, Stoffers D, Wolters ECh, Stam CJ, Berendse HW. MEG resting state functional connectivity in Parkinson's disease related dementia. J Neural Transm 2009;116:193-202.

Brown LM, Schinka JA. Development and initial validation of a 15-item informant version of the Geriatric Depression Scale. Int $\mathrm{J}$ Geriatr Psychiatry 2005;20:911-8.

Cash R, Dennis T, L'Heureux R, Raisman R, Javoy-Agid F, Scatton B. Parkinson's disease and dementia: norepinephrine and dopamine in locus ceruleus. Neurology 1987;37:42.

Caviness JN, Beach TG, Hentz JG, Shill HA, Driver-Dunckley ED, Adler CH. Association between pathology and electroencephalographic activity in Parkinson's disease. Clin EEG Neurosci 2018;49(5):321-7. 
Caviness JN, Lue LF, Hentz JG, Schmitz CT, Adler CH, Shill HA, et al. Cortical phosphorylated $\alpha$-Synuclein levels correlate with brain wave spectra in Parkinson's disease. Mov Disord 2016;31(7):1012-9.

Chapotot F, Pigeau R, Canini F, Bourdon L, Buguet A. Distinctive effects of modafinil and d-amphetamine on the homeostatic and circadian modulation of the human waking EEG. Psychopharmacology 2003;166(2):127-38.

Cummings JL, Mega M, Gray K, Rosenberg-Thompson S, Carusi DA, Gornbein J. The Neuropsychiatric Inventory: comprehensive assessment of psychopathology in dementia. Neurology 1994;44(12):2308-14.

Dauwan M, Linszen MMJ, Lemstra AW, Scheltens P, Stam CJ, Sommer IE. EEG-based neurophysiological indicators of hallucinations in Alzheimer's disease: comparison with dementia with Lewy bodies. Neurobiol Aging 2018;67:75-83.

Del Tredici K, Braak H. Dysfunction of the locus coeruleus-norepinephrine system and related circuitry in Parkinson's disease-related dementia. J Neurol Neurosurg Psychiatry 2013;84(7):774-83.

DeLong ER, DeLong DM, Clarke-Pearson DL. Comparing the areas under two or more correlated receiver operating characteristic curves: a nonparametric approach. Biometrics 1988;44(3):837-45.

Diaconescu AO, Kovacevic N, McIntosh AR. Modality-independent processes in cued motor preparation revealed by cortical potentials. Neuroimage 2008;42 (3):1255-65.

Donaghy PC, McKeith IG. The clinical characteristics of dementia with Lewy bodies and a consideration of prodromal diagnosis. Alzheimers Res Ther 2014;6(4):46.

Donaghy PC, Taylor JP, O'Brien JT, Barnett N, Olsen K, Colloby SJ, et al. Neuropsychiatric symptoms and cognitive profile in mild cognitive impairment with Lewy bodies. Psychol Med 2018;48(14):2384-90.

Dubois B, Slachevsky A, Litvan I, Pillon B. The FAB: a Frontal Assessment Battery at bedside. Neurology 2000;55(11):1621-6.

Dugger BN, Murray ME, Boeve BF, Parisi JE, Benarroch EE, Ferman TJ, et al. Neuropathological analysis of brainstem cholinergic and catecholaminergic nuclei in relation to rapid eye movement (REM) sleep behaviour disorder. Neuropathol Appl Neurobiol 2012.

Fahn S, Marsden CD, Calne DB, Goldstein M, editors. Recent developments in Parkinson's disease, vol. 2. Florham Park, NJ. Macmillan Health Care Information; 1987. p. 153-63, 293-304

Fantini ML, Gagnon JF, Petit D, Rompre S, Decary A, Carrier J, et al. Slowing of electroencephalogram in rapid eye movement sleep behavior disorder. Ann Neurol 2003;53:774-80.

Fünfgeld EW. Computerised brain electrical activity findings of parkinson patients suffering from hyperkinetic side effects (hypersensitive dopamine syndrome) and a review of possible sources. J Neural Transm Suppl 1995;46:351-65.

Halder T, Talwar S, Jaiswal AK, Banerjee A. Quantitative evaluation in estimating sources underlying brain oscillations using current source density methods and beamformer approaches. eNeuro 2019;6(4). ENEURO.0170-19.201.

Hughes SW, Crunelli V. Thalamic mechanisms of EEG alpha rhythms and their pathological implications. Neuroscientist 2005;11:357-72.

Inoue Y, Sasai T, Hirata K. Electroencephalographic finding in idiopathic REM sleep behavior disorder. Neuropsychobiology 2015;71(1):25-33.

Iranzo A, Isetta $\mathrm{V}$, Molinuevo JL, Serradell $\mathrm{M}$, Navajas $\mathrm{D}$, Farre $\mathrm{R}$, et al. Electroencephalographic slowing heralds mild cognitive impairment in idiopathic REM sleep behavior disorder. Sleep Med 2010;11:534-9.

Jackson CE, Snyder PJ, Khachaturian ZS, Brookmeyer R, Johnson E, Ziegler-Graham K, et al. Electroencephalography and event-related potentials as biomarkers of mild cognitive impairment and mild Alzheimer's disease. Alzheimers Dement 2008;4:S137-43.

James LM, Iannone R, Palcza J, Renger JJ, Calder N, Cerchio K, et al. Effect of a novel histamine subtype-3 receptor inverse agonist and modafinil on EEG power spectra during sleep deprivation and recovery sleep in male volunteers. Psychopharmacology 2011;215:643-53.

Janzen J, van't Ent D, Lemstra AW, Berendse HW, Barkhof F, Foncke EM. The pedunculopontine nucleus is related to visual hallucinations in Parkinson's disease: preliminary results of a voxel-based morphometry study. J Neurol 2012;259(1):147-54.

Jia H, Yu D. Attenuated long-range temporal correlations of electrocortical oscillations in patients with autism spectrum disorder. Dev Cogn Neurosci 2019;30(39):100687.

Jones BE, Harper ST, Halaris AE. Effects of locus coeruleus lesions upon cerebral monoamine content, sleep-wakefulness states and the response to amphetamine in the cat. Brain Res 1977;124(3):473-96.

Kai T, Asai Y, Sakuma K, Koeda T, Nakashima K. Quantitative electroencephalogram analysis in dementia with Lewy bodies and Alzheimer's disease. J Neurol Sci 2005;237(1-2):89-95.

Kamei S, Morita A, Serizawa K, Mizutani T, Hirayanagi K. Quantitative EEG analysis of executive dysfunction in Parkinson disease. J Clin Neurophysiol 2010;27:193-7.

Klimesch W, Doppelmayr M, Russegger H, Pachinger T, Schwaiger J. Induced alpha band power changes in the human EEG and attention. Neurosci Lett 1998;244 (2):73-6.

Klimesch W, Doppelmayr M, Schimke H, Pachinger T. Alpha frequency, reaction time, and the speed of processing information. J Clin Neurophysiol 1996; 13:511-8.

Klimesch W. EEG alpha and theta oscillations reflect cognitive and memory performance: a review and analysis. Brain Res Brain Res Rev 1999;29:169-95.

Kovacevic N, McIntosh AR. Groupwise independent component decomposition of EEG data and partial least square analysis. Neuroimage 2007;35(3):1103-12.
Lehmann D, Ozaki H, Pal I. EEG alpha map series: brain micro-states by spaceoriented adaptive segmentation. Electroenceph Clin Neurophysiol 1987;67:271-88.

Liu Q, Ganzetti M, Wenderoth N, Mantini D. Detecting large-scale brain networks using EEG: impact of electrode density, head modeling and source localization. Front Neuroinform 2018;12:4.

Lörincz ML, Crunelli V, Hughes SW. Cellular dynamics of cholinergically induced alpha $(8-13 \mathrm{~Hz})$ rythms in sensory thalamic nuclei in vitro. J Neurosci 2008;28:660-71.

Lorincz ML, Kékesi KA, Juhász G, Crunelli V, Hughes. Temporal framing of thalamic relay-mode firing by phasic inhibition during the alpha rythm. Neuron 2009;63:683-96.

Marino M, Liu Q, Brem S, Wenderoth N, Mantini D. Automated detection and labeling of high-density EEG electrodes from structural MR images. J Neural Eng 2016;13(5):056003.

Massicotte-Marquez J, Carrier J, Decary A, Mathieu A, Vendette M, Petit D, et al. Slow-wave sleep and delta power in rapid eye movement sleep behavior disorder. Ann Neurol 2005;57:277-82.

Massironi G, Galluzzi S, Frisoni GB. Drug treatment of REM sleep behavior disorders in dementia with Lewy bodies. Int Psychogeriatr 2003;15(4):377-83.

McKeith IG, Boeve BF, Dickson DW, Halliday G, Taylor JP, Weintraub D, et al. Diagnosis and management of dementia with Lewy bodies: fourth consensus report of the DLB Consortium. Neurology 2017;89(1):88-100.

McKeith IG, Dickson DW, Lowe J, Emre M, O'Brien JT, Feldman H, et al. Diagnosis and management of dementia with Lewy bodies: third report of the DLB consortium. Neurology 2005:65:1863-72.

McKeith IG, Galasko D, Kosaka K, Perry EK, Dickson DW, Hansen LA, et al. Consensus guidelines for the clinical and pathologic diagnosis of dementia with Lewy bodies (DLB): report of the consortium on DLB international workshop. Neurology 1996;47(5):1113-24.

McKhann G, Drachman D, Folstein M, Katzman R, Price D, Stadlan EM. Clinical diagnosis of Alzheimer's disease: report of the NINCDSADRDA work group under the auspices of department of health and human services task force on alzheimer's disease. Neurology 1984;34:939-44.

Melgari JM, Curcio G, Mastrolilli F, Salomone G, Trotta L, Tombini M, et al. Alpha and beta EEG power reflects L-dopa acute administration in parkinsonian patients. Front Aging Neurosci 2014;6:302.

Michel CM, Koenig T. EEG microstates as a tool for studying the temporal dynamics of whole-brain neuronal networks: a review. Neuroimage 2018;180:577-93.

Michel CM, Lantz G, Spinelli L, de Peralta RG, Landis T, Seeck M. 128-Channel EEG source imaging in epilepsy: clinical yield and localization precision. J Clin Neurophysiol 2004;21(2):71-83.

Moretti DV, Babiloni C, Binetti G, Cassetta E, Dal Forno G, Ferreric F, et al. Individual analysis of EEG frequency and band power in mild Alzheimer's disease. Clin Neurophysiol 2004;115(2):299-308.

Moretti DV, Babiloni F, Carducci F, Cincotti F, Remondini E, Rossini PM, et al. Computerized processing of EEG-EOG-EMG artifacts for multicentirc studies in EEG oscillations and event-related potentials. Int J Pshycophysiol 2003;47 (3):199-216.

Pascual-Marqui RD. Discrete, 3D distributed, linear imaging methods of electric neuronal activity. Part 1: exact, zero error localization. 2007 arXiv:0710.3341 [math-ph] Available at: http://arxiv.org/pdf/0710.3341 [accessed November 19, 2015].

Pfurtscheller G, Lopes da Silva FH. Event-related EEG/MEG synchronization and desynchronization: basic principles. Clin Neurophysiol 1999;110:1842-57.

Pugnetti L, Baglio F, Farina E, Alberoni M, Calabrese E, Gambini A, et al. EEG evidence of posterior cortical disconnection in PD and related dementias. Int J Neurosci 2010;120:88-98.

Rodrigues Brazete J, Montplaisir J, Petit D, Postuma RB, Bertrand JA, Genier Marchand D, et al. Electroencephalogram slowing in rapid eye movement sleep behavior disorder is associated with mild cognitive impairment. Sleep Med 2013;14:1059-63.

Saletu M, Anderer P, Saletu-Zyhlarz GM, Mandl M, Arnold O, Zeitlhofer J, et al. EEGtomographic studies with LORETA on vigilance differences between narcolepsy patients and controls and subsequent double-blind, placebo-controlled studies with modafinil. J Neurol 2004;251(11):1354-63.

Saletu M, Anderer P, Semlitsch HV, Saletu-Zyhlarz GM, Mandl M, Zeitlhofer J, et al. Low-resolution brain electromagnetic tomography (LORETA) identifies brain regions linked to psychometric performance under modafinil in narcolepsy. Psychiatry Res 2007;154(1):69-84.

Samogin J, Liu Q Marino M, Wenderoth N, Mantini D. Shared and connectionspecific intrinsic interactions in the default mode network. Neuroimage 2019;200:474-81.

Serizawa K, Kamei S, Morita A, Hara M, Mizutani T, Yoshihashi H, et al. Comparison of quantitative EEGs between Parkinson disease and age-adjusted normal controls. J Clin Neurophysiol 2008;25:361-6.

Steriade M, Llinás RR. The functional states of the thalamus and the associated neuronal interplay. Physiol Rev 1988;68(3):649-742.

Stylianou M, Murphy N, Peraza LR, Graziadio S, Cromarty R, Killen A, et al. Quantitative electroencephalography as a marker of cognitive fluctuations in dementia with Lewy bodies and an aid to differential diagnosis. Clin Neurophysiol 2018;129(6):1209-20.

Vermeiren Y, Deyn De. Targeting the norepinephrinergic system in Parkinson's disease and related disorders: the locus coeruleus story. Neurochem Int 2017;102:22-32. 
Walker MP, Ayre GA, Cummings JL, Wesnes K, McKeith IG, O’Brien JT, et al. The clinician assessment of fluctuation and the one day fluctuation assessment scale. Two methods to assess fluctuating confusion in dementia. Br J Psychiatry 2000a; 177:252-6.

Walker MP, Ayre GA, Perry EK, Wesnes K, McKeith IG, Tovee M, et al. Quantification and characterization of fluctuating cognition in dementia with Lewy bodies and Alzheimer's disease. Dement Geriatr Cogn Disord 2000b;11(6):327-35.
Weisman D, Cho M, Taylor C, Adame A, Thal LJ, Hansen LA. In dementia with Lewy bodies, Braak stage determines phenotype, not Lewy body distribution. Neurology 2007;69(4):356-9.

Yeomans JS. Muscarinic receptors in brain stem and mesopontine cholinergic arousal functions. Handb Exp Pharmacol 2012;208:243-59.

Zweig RM, Cardillo JE, Cohen M, Giere S, Hedreen JC. The locus ceruleus and dementia in Parkinson's disease. Neurology 1993;43:986. 